\title{
A constitutive model for crushed salt
}

\author{
S. Olivella* ${ }^{* \dagger}$ and A. Gens \\ E.T.S. de Ingenieros de Caminos, Canales y Puertos, Universidad Politecnica de Catalunya, Barcelona, Spain
}

\begin{abstract}
SUMMARY
A constitutive model for crushed salt is presented in this paper. A creep constitutive model is developed first and compared with test results. The constitutive model presented here concentrates on creep deformation because saline media behave basically in a ductile and time-dependent way. An idealized geometry is used as a common framework to obtain stress-strain macroscopic laws based on two deformation mechanisms: fluid-assisted diffusional transfer creep and dislocation creep. The model is able to predict strain rates that compare well with results from laboratory tests under isotropic and oedometric conditions. Macroscopic laws are written using a non-linear viscous approach, which incorporates also a viscoplastic component, based on critical state theory. The viscoplastic term is intended for non-creep deformation mechanisms such as grain reorganization and crushing. Copyright (C) 2002 John Wiley \& Sons, Ltd.
\end{abstract}

\section{INTRODUCTION}

An introduction to saline media, in general, precedes a description of deformation processes in rock salts and porous aggregates.

\subsection{Background}

Salt rock is considered as a possible geological medium for underground radioactive waste disposal. Seals in salt-rock caverns for isolating purposes are designed to be made of porous crushed salt with different degrees of compaction, i.e. with different initial porosities $\phi_{0}$. Backfilling of cavities is carried out with slightly compacted crushed salt $\left(\phi_{0}=0.3-0.4\right)$ while seals (also called dams) can be built with salt bricks with a higher degree of compaction $\left(\phi_{0}=0.1-0.2\right)$. Figure 1 shows crushed salt before and after compaction. Temperature and humidity content are crucial variables in the mechanical behaviour of these aggregates.

The study of these seals requires the development of new approaches. The main reason is that fundamental processes taking place in salt are less relevant in other geological materials or take place much slowly. While salt rock behaviour is relatively well understood, the behaviour of porous salt aggregates is much less known. Therefore, research activity has recently focused

*Correspondence to: S. Olivella, E.T.S. de Ingenieros de Caminos, Canales y Puertos, Universidad Politecnica de Catalunya, Barcelona, Spain

†E-mail: sebastia.olivella@upc.es 


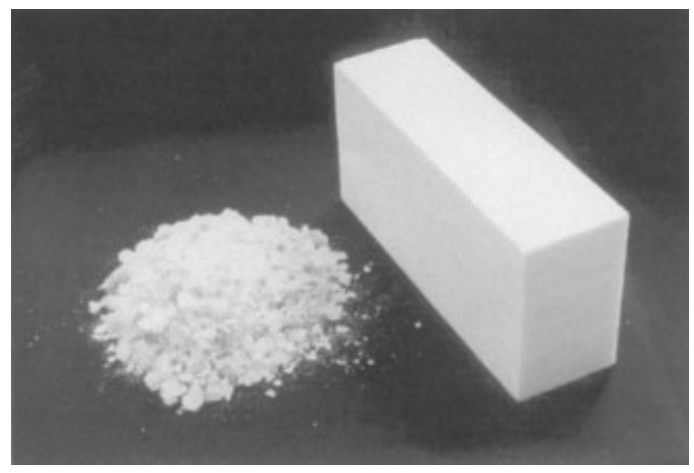

Figure 1. Crushed salt before and after compaction.

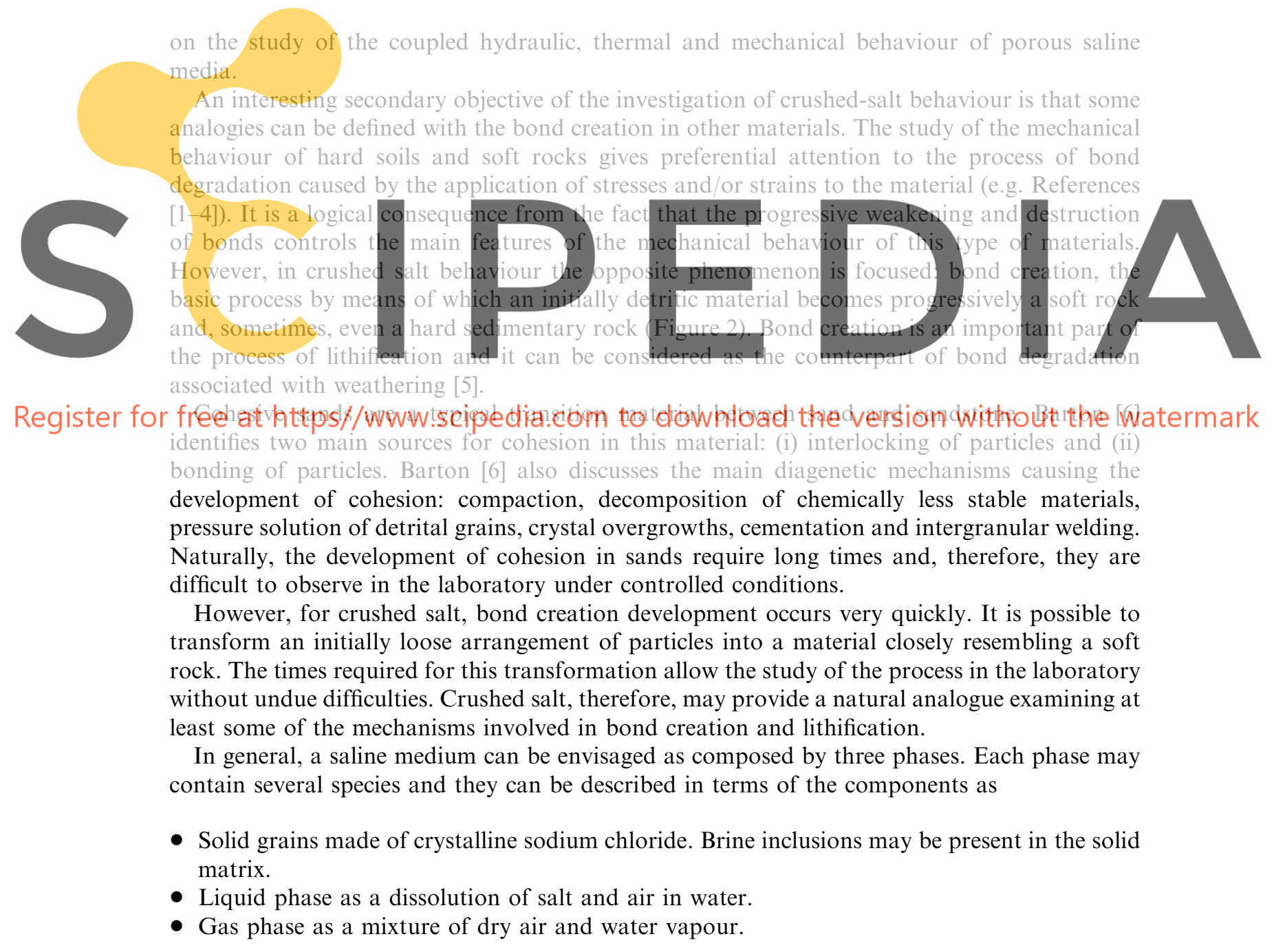




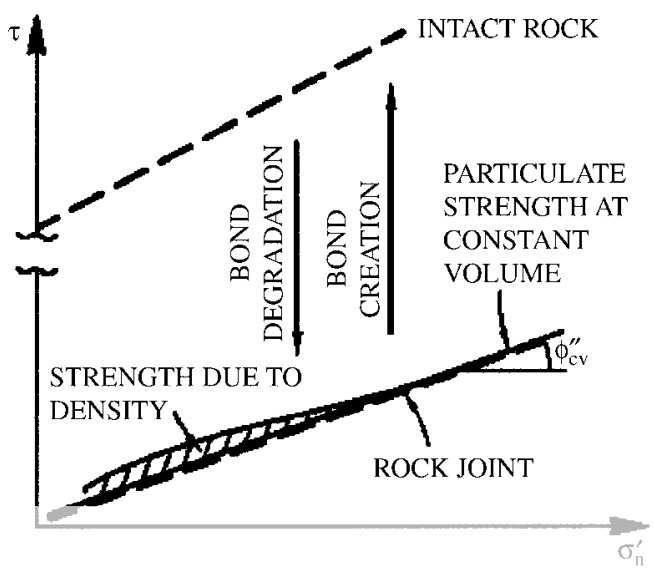

Figure 2. The transition between soil and rock (adapted from Reference [5]).

The behaviour of porous salt aggregates is complex. As mentioned above, phase interaction involves processes that take place in much slower time scale in other geological media. For instance, dissolution and precipitation of salt implies major differences with other materials. In this regard, variations of porosity may take place induced, for instance, by temperature

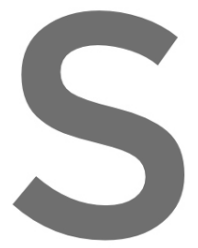
differences [7]. From deformation. Presence precipitation at the $\mathrm{p}$ coupling between $m$ process mechanics salt creep is strongly $d$
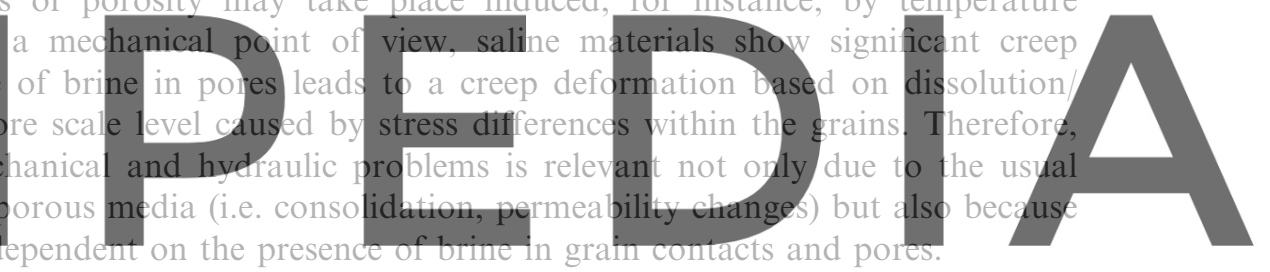

On the other hand, there exist strong coupling effects between thermal phenomena and

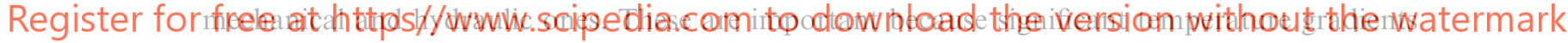
exist in problems of waste disposal so that the medium is under non-isothermal conditions. Apart from the classical couplings (e.g. thermal expansion), it should be noticed that the following processes depend strongly on temperature:

- Creep deformation of salt rock. Creep strain rates are highly dependent on temperature.

- Solubility of salt in water. The concentration of a saturated dissolution is a function of temperature.

- Water vapour concentration and motion in gas phase. Consideration of phase change requires enthalpy balance.

- Viscous motion of fluids. Viscosity of fluids depends on temperature.

The study of a medium of this type, leads to a physico-mathematical approach in the field of multiphase-multicomponent flow under non-isothermal conditions including deformation. Three main directions of investigation, theoretical and computational, have been followed in order to develop a comprehensive approach:

- Formulation of the balance equations for mass, momentum and energy.

- Development or adaptation of constitutive laws.

- Numerical implementation of balance equations together with constitutive laws. 
The formulation of the balance equations can be found in Reference [8]. In this work, partial differential equations were established paying specific attention to the relevant processes that take place in the saline media.

\subsection{Deformation of salt rock and salt aggregates}

A low-porosity saline rock subject to a confining stress deforms in a ductile way if a deviatoric stress state is applied. The strain rates that are developed are, in general, a non-linear function of deviatoric stress and depend strongly on medium temperature [9]. Under low confining stress states, dilatancy appears and the material becomes more brittle as confining stress decreases or strain rate increases [10]. Strength is a function of confining stress, strain rate and temperature. Strength tests under different stress states have been carried out by Hunsche and Albrecht [11] in a true triaxial cell. Viscoelasticity and viscoplasticity provide an adequate framework for characterizing the behaviour of rock salt. The existing constitutive models range from a simple power law [12] which stands for history-independent deviatoric strains, to more complex models $[12,13]$, which include other features such as dilatancy or hardening/softening behaviour. In general, these models were developed to analyse saline media with a small or negligible porosity, i.e. salt rocks.

The study of the behaviour of porous salt aggregates is gaining interest, mainly due to the possible use of such materials to backfill openings in radioactive waste repositories. Early studies of crushed sat behaviour adopted, in some cases, an approach similar to that used in soil

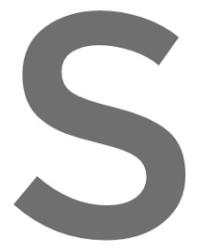
mechanics $[14,15]$. P strain rates. More recent very interesting model et al. [16-18].

They have explained
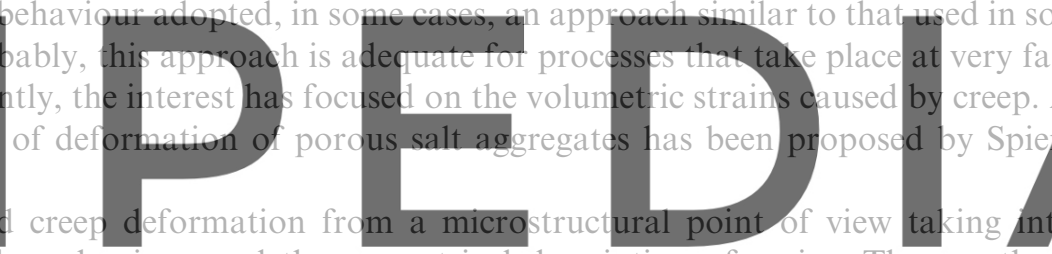

account basic physical mechanisms and the geometrical description of grains. These authors proposed a model for volumetric isotropic deformation, Other authors [19] based their

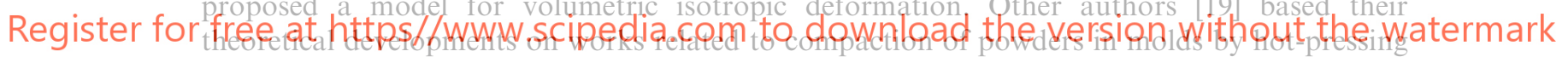

[20], a field in which several models have been developed.

A mechanical constitutive model to represent the creep behaviour of porous salt aggregates has been developed, and it is described in this paper. The basic creep model was firstly presented by Olivella et al. [21-23]. It has been used for modelling in several applications related to saline formations. Here, the combination of the basic creep model with viscoplasticity is also included.

\subsection{Microstructural observations}

A direct evidence of the progress of compaction and bond development is provided by microscope observation of the microfabric of the materials. Figure 3 presents a sequence of micrographs in which the transition from the initial loose state to a significantly denser material can be observed. The material was prepared from analytical-grade $\mathrm{NaCl}$ powder, sieved into prescribed grain-size fractions. Grading was, therefore, very uniform. Particle interlocking, crystal overgrowth and the appearance of new contacts arising from pressure solution can be discerned.

However, the main practical interest lies in checking whether, by continuing compaction further, crushed salt may eventually resemble rock salt. In that respect, the similarity of the two 


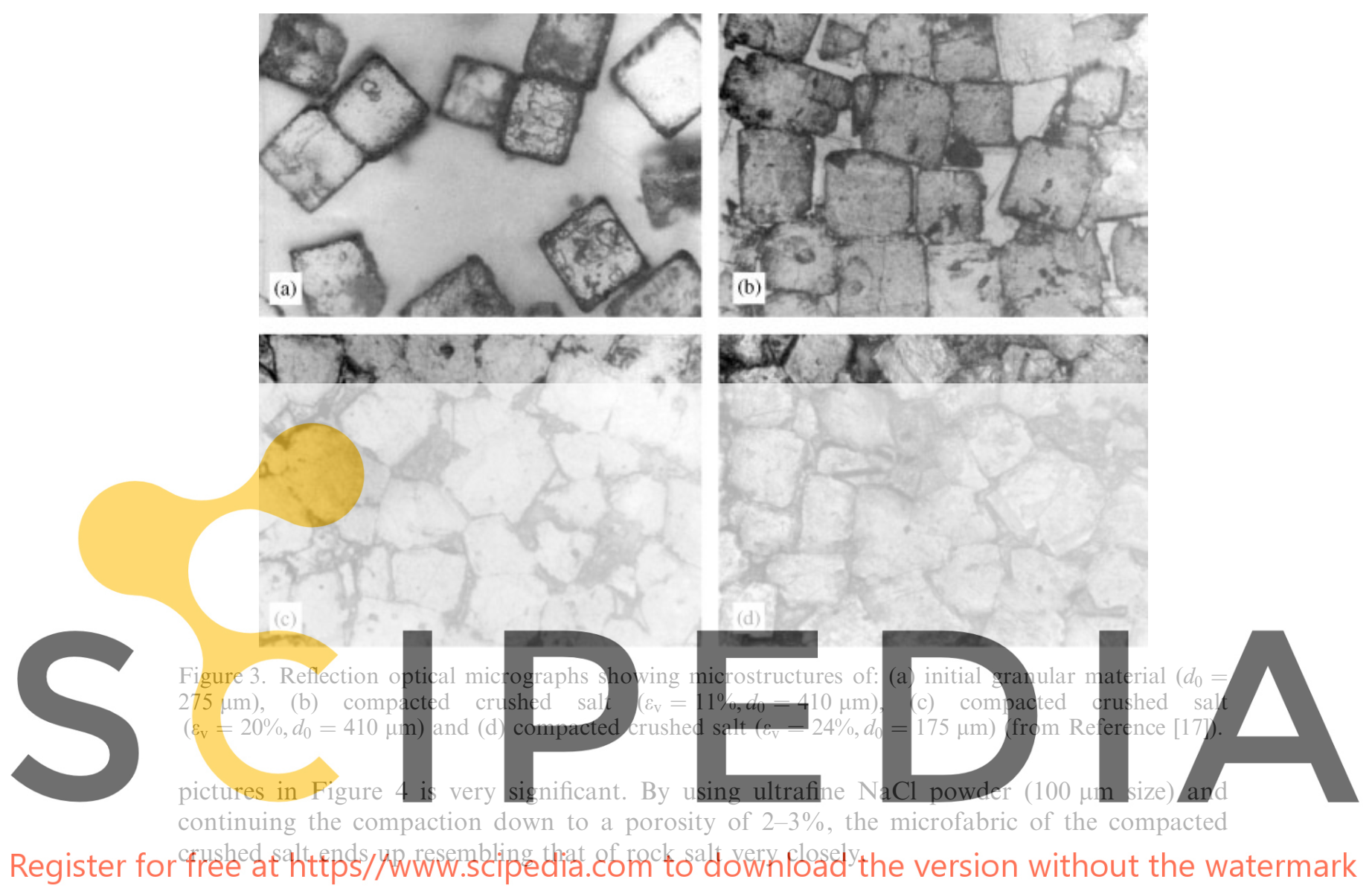

\section{CONSTITUTIVE MODEL FOR CREEP DEFORMATION OF POROUS SALT AGGREGATES}

A general model for the creep behaviour of salt aggregates was required to undertake fully coupled analysis of salt-based sealing systems. The most relevant mechanisms of deformation in saline materials are linked with creep or ductile deformation. Two main goals had to be achieved: good predictive capabilities for the stress-strain processes that take place in real conditions, and, implementation in a coupled numerical tool. In order to ensure the first objective, it is necessary to compare the model with test results. For the second one, the equations theoretically developed should be transformed into a viscoelastic or viscoplastic framework suitable for incorporation into a numerical formulation.

\subsection{Most relevant deformation mechanisms in salt aggregates}

In contrast to other rocks, rock salt shows creep deformation at room temperature. Other rocks require very high temperatures and degree of confinement to behave with such strain rates, 

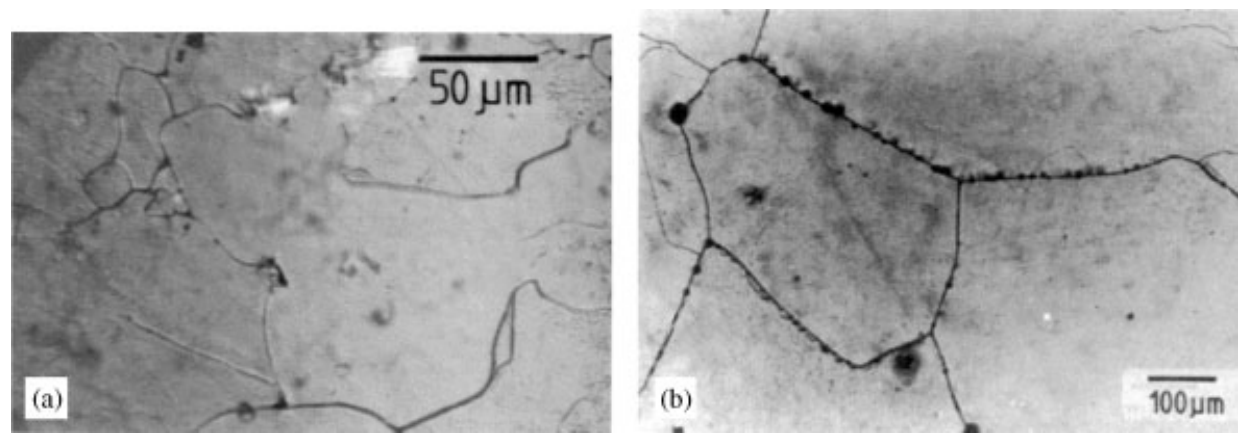

Figure 4. (a) Reflection optical micrograph of ultrafine highly compacted $\mathrm{NaCl}$ powder (from Reference [17]) and (b) Micrograph of rock salt (from Reference [16]).
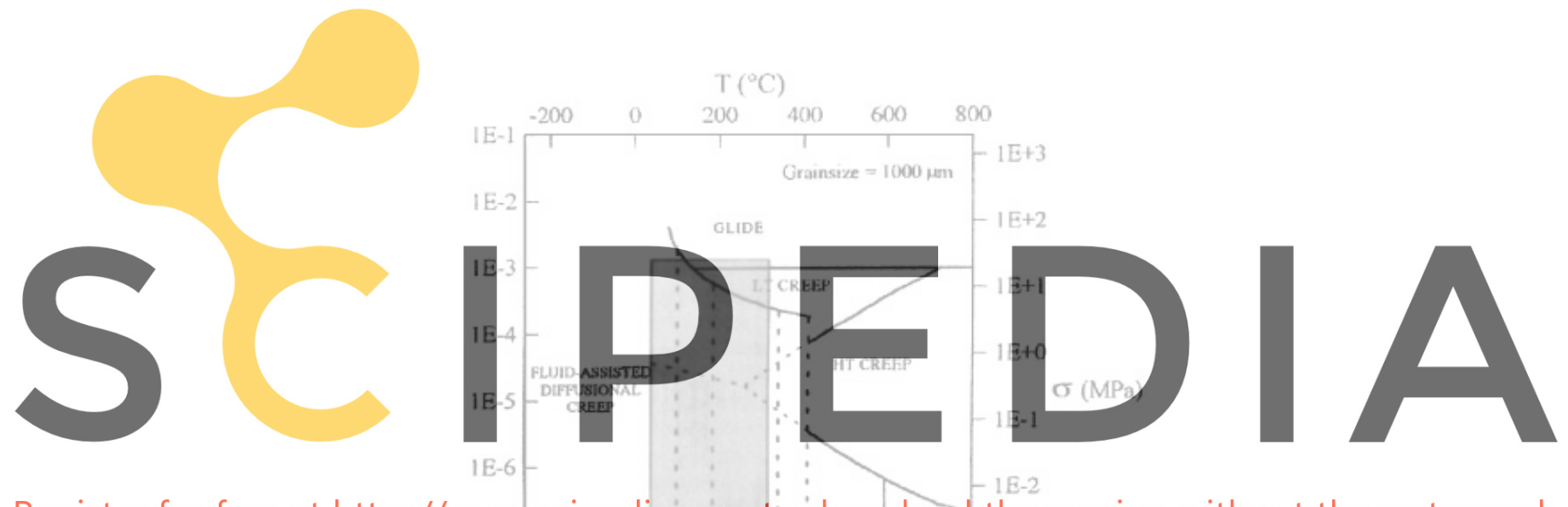

Register for free at https//www.scipediaicom to download the version without the watermark

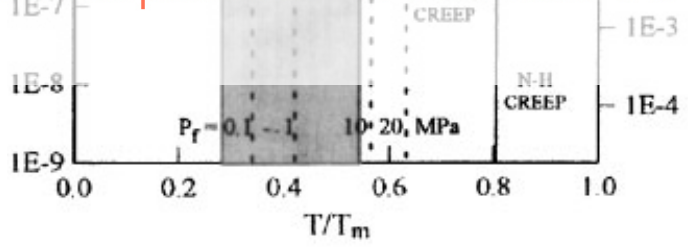

Liquid/Vapor transition

Probable repository conditions

Figure 5. Stress temperature deformation mechanism map.

otherwise they deform in a brittle manner. In Figure 5 a stress/temperature deformation mechanism map is presented. This map shows that depending on temperature and stress level, creep deformation is caused by different mechanisms. Glide and climb (low and high temperature) mechanisms are related to dislocation theory, hence, they can be seen as intracrystalline mechanisms. At high temperatures $\left(T / T_{\mathrm{m}}\right.$ above 0.6 , where $T_{\mathrm{m}} \cong 800^{\circ} \mathrm{C}$ is melting temperature) solid diffusion mechanisms may occur. At low stresses and temperatures, 
the most important mechanism of creep deformation is based on fluid-assisted diffusion. This mechanism is intercrystalline because diffusion takes place along grain boundaries. In the map, the probable conditions for stresses and temperatures in backfills and seals of repositories are indicated.

The constitutive model has been based on the following two mechanisms of deformation:

Fluid-assisted diffusional transfer deformation (FADT); and dislocation creep (DC) deformation.

that are expected to play a significant role in repository conditions. A description of these two deformation mechanisms follows below in Figures 6 and 7.

FADT refers to the mechanism of deformation based on the migration of salt through the liquid phase present in pores. This migration of salt is developed from contacts to contacts or from contacts to pores. The driving force for diffusion is the gradient of chemical potential induced by differences in contact stresses.

DC refers to intracrystalline mechanisms explained through dislocation theory. The crystalline net is not perfect because dislocations and voids occur. A deviatoric stress applied to a crystalline body causes movement of such imperfections which are macroscopically seen as deformations.
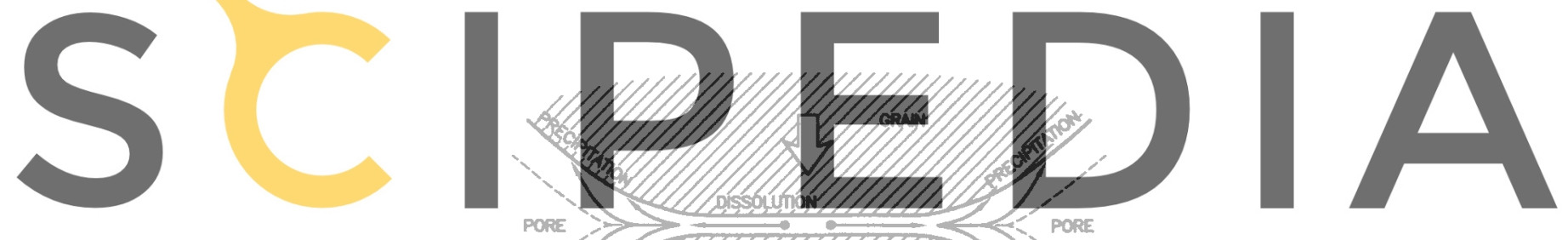

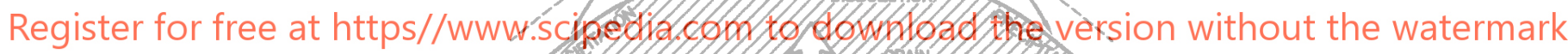

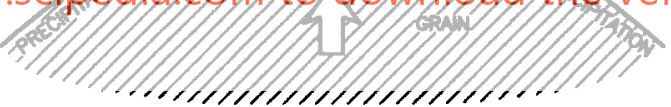

Figure 6. FADT creep.

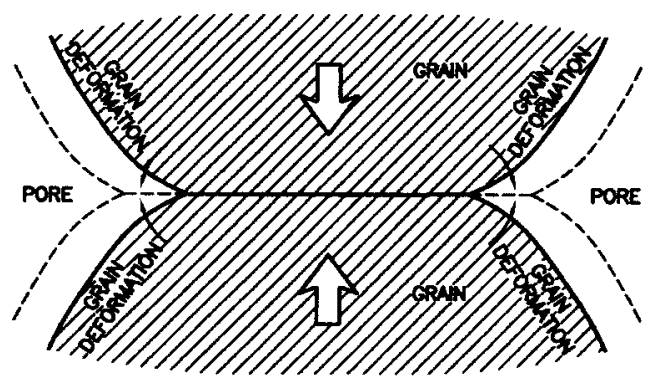

Figure 7. DC mechanism. 
As mentioned above, solid diffusion mechanisms are only important under high temperatures and low-stress states, hence these mechanisms are not considered relevant in this context. They can be neglected because FADT and DC are the dominant under repository conditions.

\subsection{Idealized geometry for grains and pores}

It has been found useful to base the development of the constitutive model on an idealized geometry incorporating some features of the real microstructure of the porous salt aggregates.

The idealized microstructure arrangement is composed by a regular arrangement of polyhedrons (Figure 8). This geometry is used as a basis for calculating strain rates and to obtain macroscopic laws. In order to provide a common framework for both mechanisms, the same idealized geometry is used. The microstructure of salt granular aggregates has been observed by Spiers et al. [17,24], and these authors have shown micrographs where regular cubic forms can be clearly distinguished. It must be recalled that sodium chloride belongs to the crystallographic cubic system. This regular form of grains has led to idealize the geometry using polyhedral forms as simple as possible. An idealized geometry for grains and pores could be based, alternatively, on spherical particles, as for instance, Spiers et al. [17] have done.

In order to simplify the subsequent mathematical developments, two hypotheses concerning the idealized geometry are adopted:

- The principal directions of the macroscopic stress state are normal to the faces of the
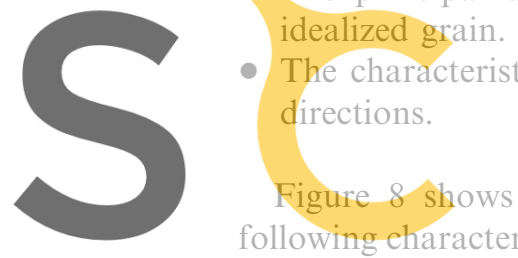

- The characteristic directions.

Figure 8 shows following characteristic sizes: $\boldsymbol{d}$, grain size and
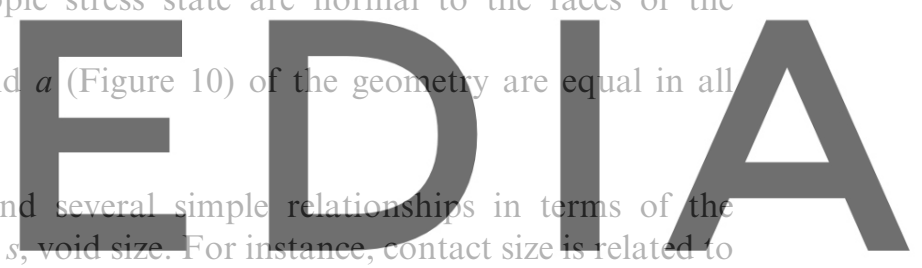

Register for free at https//www.scipedia.com to download the version without the watermark

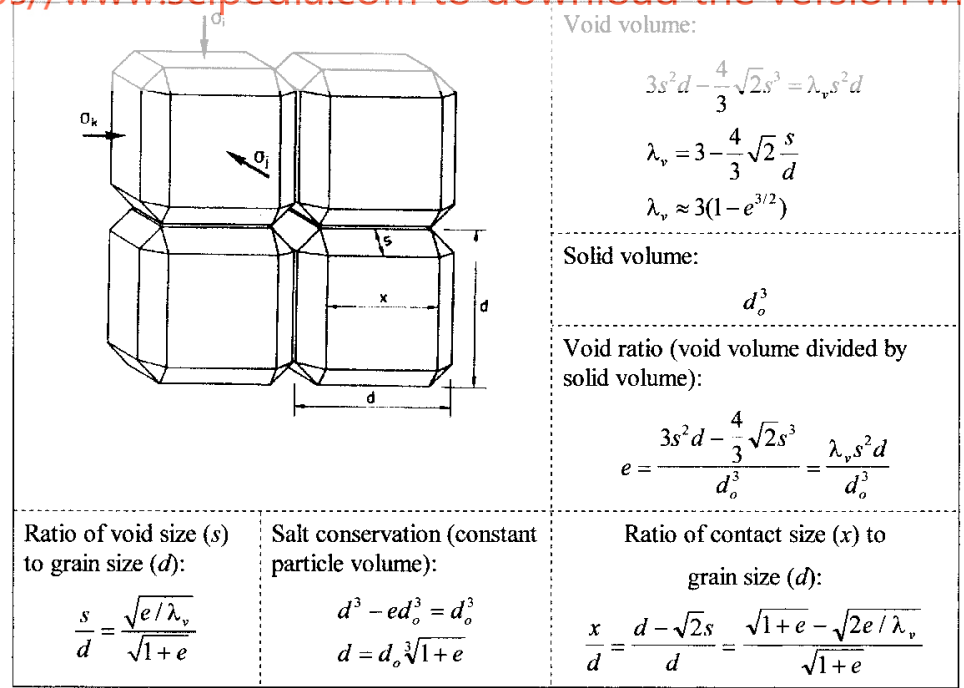

Figure 8. Idealized geometry of grains and pores used to develop a creep model. 
them as $x=d-\sqrt{2} s$. Void volume, void ratio and the ratio of stress concentration can be computed (Figure 8) in an easy way.

It is convenient to write every geometrical variable as a function of void ratio. In this way the introduction of these variables in equations, produces dependencies only on void ratio and not on the characteristic sizes which are difficult to determine in a real granular aggregate. However, this three-dimensional geometry leads to somewhat complex expressions, e.g. relative pore size is $s / d=\sqrt{e / \lambda_{\mathrm{v}}} / \sqrt{1+e}$ with $\lambda_{\mathrm{v}}=3-(4 / 3) \sqrt{2}(s / d)$, which is not explicit. Unnecessary complexity of the equations would appear if this form is maintained. To overcome this practical difficulty, $\lambda_{\mathrm{v}}$ has been modified to $\lambda_{\mathrm{v}} \cong 3\left(1-e^{3 / 2}\right)$. The modified function has the following advantages: simplifies the resulting relationships, for low values of $e$ converges to the theoretical expression, and when comparing the model with experimental results for different values of $e$, it has exhibited a better performance than the algebraically derived one. The use of a modified $\lambda_{\mathrm{v}}$ does not imply any loss of generality in the geometrical relationships. The contact size and the pore-size functions, which are key functions in the model, are represented in Figure 9 as a function of void ratio. It can be observed from this plot that for these relationships a void ratio of approximately $e=0.78$ is an upper bound of validity of the equations. Higher values of void ratio would produce contacts with negative size.

\section{The main features of this approximated idealization of the microstructure are}

- All geometrical variables (Figure 8) are only functions of void ratio (e).
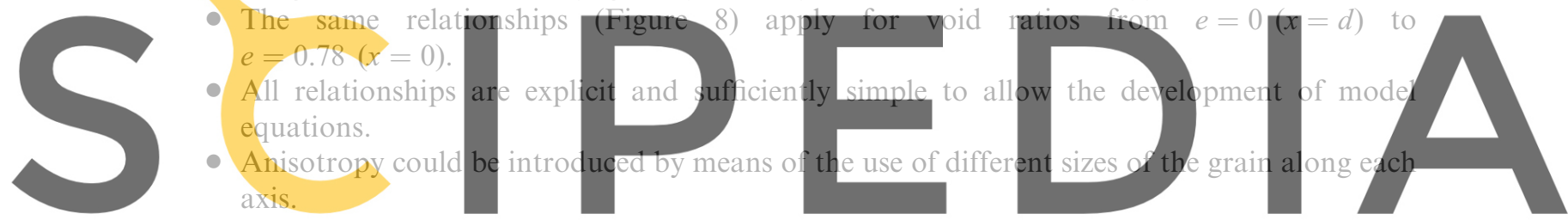

Register for free at https//www.scipedia.com to download the version without the watermark

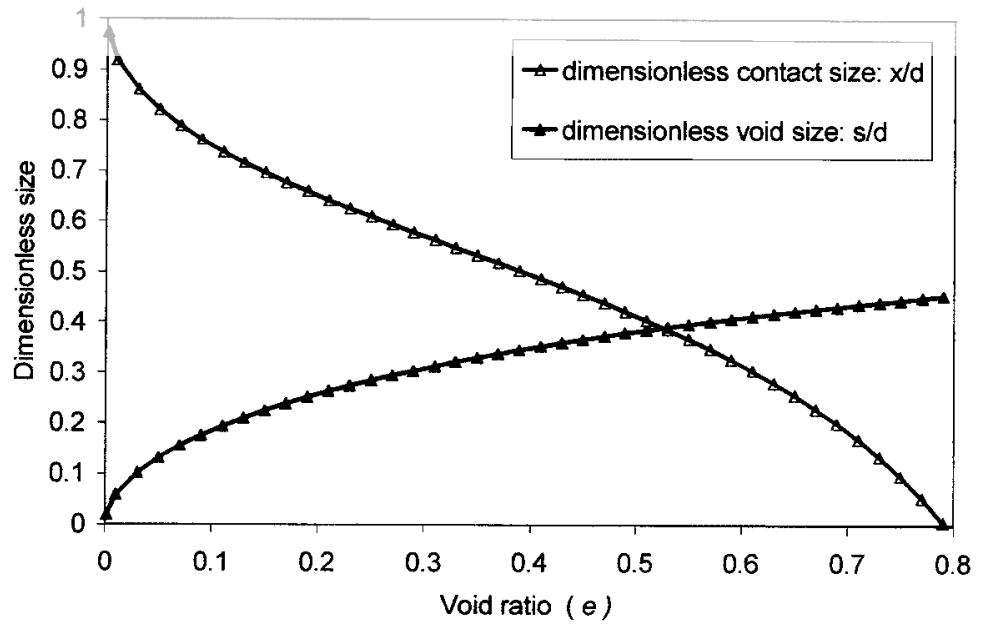

Figure 9. Variation of contact size $(x / d)$ and void size $(s / d)$ with void ratio using the modified function (empirical). 


\subsection{FADT mechanism}

The first mechanism that was investigated is FADT. It involves basically the deformation of grains by dissolution/migration/precipitation of salt from zones of stress concentration to zones of lower stress levels. Since salt migration takes place through the liquid phase, this mechanism will only operate if brine is present. For porous salt aggregates subjected to relatively confined conditions, salt migration takes place from contacts to pores causing reduction of pore volume. This mechanism of deformation has been extensively studied by Spiers et al. [16-18,24]. Their ideas have been followed in the development of a constitutive model. Experimental data from these authors have been useful to validate model predictions.

The medium is considered saturated with brine at a pressure $P_{\ell}$ pressure and macroscopic stresses $\sigma_{i}, \sigma_{j}, \sigma_{k}$ are applied externally (principal stress tensor components).

A macroscopic model for FADT can be obtained if the following hypotheses are considered:

- Chemical potential $(\mu)$ is the driving force for dissolution/transport/precipitation of salt.

- In the absence of flow, the dissolution/transport/precipitation is diffusion controlled.

Dissolution and precipitation take place with negligible chemical potential losses compared to diffusion.

- Chemical potential is the same in the solid and in the solution in contact with it.

- Differences in chemical potential are induced by differences in normal stress on the solid.

- A fluid film of thickness $a$ exists in the contacts.
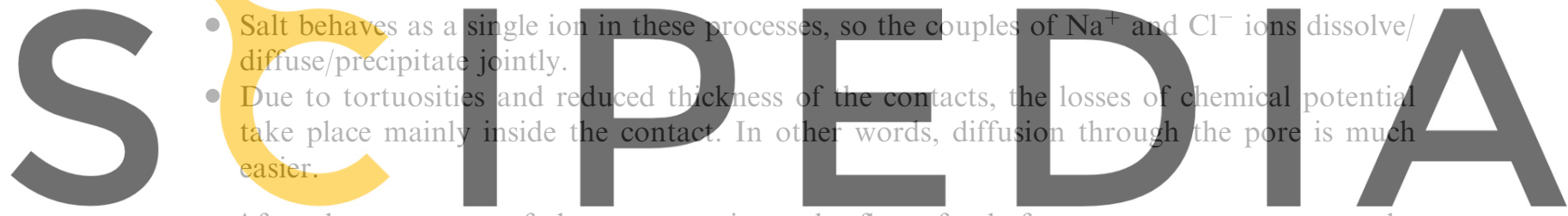

After the statement of these assumptions, the flux of salt from contact to pores can be Register fortained and then related to macroscopic deformations. This will be done in five.steps. Piffusive

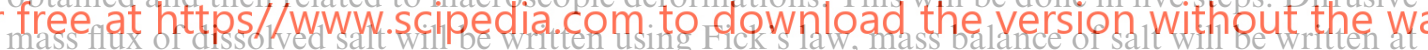

the grain scale, chemical potential gradients will be estimated as a function of stress

concentration on contacts, strain rates computed from grain size variations and the model will be generalized to a tensorial form.

2.3.1. Diffusive mass flux of dissolved salt. Fick's law allows to express the diffusive mass flux of salt in solution as [25]

$$
i=-D \nabla \omega=-\tau \rho_{\ell} D_{\mathrm{m}} \frac{\omega m}{R T} \nabla \mu
$$

where $\omega$ is the mass fraction of salt in dissolution and $D$ is the coefficient of effective diffusion expressed as $D=\tau \rho_{\ell} D_{\mathrm{m}}$, where $\tau$ is the tortuosity coefficient, $\rho_{\ell}$ is the liquid density and $D_{\mathrm{m}}$ is the molecular diffusion coefficient. The second equivalence in (1) has been obtained from the definition of chemical potential in terms of solute concentrations:

$$
\mu=\mu_{0}+R T \log (\gamma \chi), \quad \frac{\nabla \mu}{R T}=\frac{\nabla \chi}{\chi}=\frac{\nabla \omega}{m \omega}, \quad m=M_{\mathrm{w}}\left(\frac{\omega}{M_{\mathrm{h}}}+\frac{1-\omega}{M_{\mathrm{w}}}\right)
$$

where $\mu_{0}$ is a reference value for chemical potential, $R$ is the constant of gases $(8.314 \mathrm{~J} / \mathrm{mol} / \mathrm{K})$, $T$ is the absolute temperature, $\chi$ is the mole fraction, $\gamma$ is the activity coefficient and $M_{\mathrm{h}}$ and $M_{\mathrm{w}}$ are molecular weights of salt and water, respectively. 
2.3.2. Mass balance of salt at the grain scale. The mass flux of salt will cause a reduction of the grain and pore sizes $(d, s)$ while the mass should be locally conserved. Therefore, the salt mass flux through the lateral area of the fluid film in the contact must equal the salt removed from the contact. This is expressed by

$$
4 \operatorname{ax}|i|=\rho_{\mathrm{s}} x^{2} \frac{\mathrm{d} d}{\mathrm{~d} t}
$$

where | $\mid$ is the module, $\mathrm{d} d / \mathrm{d} t$ is the time variation of $d, \rho_{\mathrm{s}}$ is the solid density and $x$ is the contact length.

2.3.3. Estimation of chemical potential gradients. Since not all the dissolved salt will travel the same distance from the contact to the pore, it is assumed that the mean distance for salt diffusion through the pore is $x / 4$ (Figure 10). Then, the chemical potential gradient is approximated as

$$
\nabla \mu=\frac{\Delta \mu}{x / 4}=\frac{\mu_{\text {contact }}-\mu_{\text {pore }}}{x / 4}
$$

At this point the soil mechanics concept of effective stress will be recalled. Considering that the medium is fully saturated with brine at pressure $P_{\ell}$, this effective stress is $\sigma_{i}^{\prime}=\sigma_{i}-P_{\ell}$ and the mean effective stress can be obtained as the average of normal stresses as $p^{\prime}=\left(\sigma_{1}^{\prime}+\sigma_{2}^{\prime}+\sigma_{3}^{\prime}\right) / 3$. A saturated material will only deform when subjected to changes of effective stress, and as effective stresses tend to zero, the granular material loses any shear resistance because contacts tend to separate.

According to Spiers et al. [17] and Schutjens [24], chemical potential in contacts can be approximated as

$$
\mu_{\text {contact }}=\left(\sigma_{i}^{\prime}\right)_{\mathrm{c}} V_{\mathrm{m}}+P_{\ell} V_{\mathrm{m}}
$$

where $V_{\mathrm{m}}$ is the mole volume, and $O_{\mathrm{c}}$ indicates the contact stress (Figure 9). For the value of chemical potential at the pore, it is proposed in this paper to use

$$
\mu_{\text {pore }}=P_{\ell} V_{\mathrm{m}}+\left(p^{\prime}\right)_{\mathrm{c}}(1-f) V_{\mathrm{m}}
$$

as a modification of the usual relationship used by these authors which only contains the first term $\mu_{\text {pore }}=P_{\ell} V_{\mathrm{m}}$. As shown later, this modification allows to ensure that as pores disappear the volumetric strain rate also vanishes. In fact, the surface of the grain facing the pore progressively becomes part of the grain contact as void volume reduces. A value of $f=e^{3 / 2}$ has been adopted on an empirical basis by comparison of the predictions of the final equations of the model with experimental results.

2.3.4. Strain rate for $F A D T$. Strain rate along principal direction $(i)$ is obtained by combination of the above equations and the relationships derived from the geometry. As the size of the grain

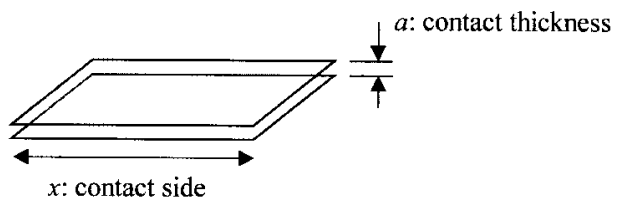

Figure 10. Scheme of the contact. 
(d) changes with time, strain rate along any principal direction $i$ can be estimated as $\left(\mathrm{d} \varepsilon_{i} / \mathrm{d} t=(1 / \mathrm{d}) \mathrm{d} d / \mathrm{d} t\right.$, where $\mathrm{d} d / \mathrm{d} t$ will be computed from Equations (3), (1) and (4) and $d$ from geometrical considerations (Figure 8 ). This leads to

$$
\begin{aligned}
\frac{\mathrm{d} \varepsilon_{i}}{\mathrm{~d} t} & =\left(\frac{\dot{d}}{d}\right)_{i}=16 B \frac{\left(\sigma_{i}^{\prime}-p^{\prime}(1-f)\right) d^{2}}{d x^{2} x^{2}}=16 \frac{B}{d_{0}^{3}} B \frac{\left(\sigma_{i}^{\prime}-p^{\prime}(1-f)\right)(1+e)}{\left(\sqrt{1+e}-\sqrt{2 e / \lambda_{\mathrm{V}}}\right)^{4}} \\
B & =\frac{\tau D_{\mathrm{m}} \rho_{\ell} m \omega V_{\mathrm{m}} a}{R T \rho_{\mathrm{s}}}
\end{aligned}
$$

in which a material parameter $B$ has been defined by compilation of physical constants. Essentially, this material parameter depends on temperature and mineral properties. The intercontact thickness $(a)$ is also included in $B$.

Once the strain rates along principal directions have been obtained, volumetric strain rate is computed as

$$
\frac{\mathrm{d} \varepsilon_{\mathrm{V}}}{\mathrm{d} t}=\frac{\mathrm{d} \varepsilon_{1}}{\mathrm{~d} t}+\frac{\mathrm{d} \varepsilon_{2}}{\mathrm{~d} t}+\frac{\mathrm{d} \varepsilon_{3}}{\mathrm{~d} t}=16 \frac{B}{d_{0}^{3}} \frac{\left(p^{\prime} f\right)(1+e)}{\left(\sqrt{1+e}-\sqrt{2 e / \lambda_{\mathrm{V}}}\right)^{4}}=\frac{p^{\prime}}{\eta_{\mathrm{v}}^{\text {FADT }}\left(e, T, d_{0}\right)}
$$

and deviatoric strain rate is obtained as

$$
\frac{\mathrm{d} e_{i}}{\mathrm{~d} t}=\frac{\mathrm{d} \varepsilon_{i}}{\mathrm{~d} t}-\frac{1 \mathrm{~d} \varepsilon_{\mathrm{V}}}{3 \mathrm{~d} t}=16 \frac{B}{d_{0}^{3}} \frac{\left(\sigma_{i}^{\prime}-p^{\prime}\right)(1+e)}{\left(\sqrt{1+e}-\sqrt{2 e / \lambda_{\mathrm{V}}}\right)^{4}}=\frac{\left(\sigma_{i}^{\prime}-p^{\prime}\right)}{\eta_{\mathrm{d}}^{\text {FADT }}\left(e, T, d_{0}\right)}
$$

The new variables $\eta_{\mathrm{v}}^{\text {FADT }}$ and $\eta_{\mathrm{d}}^{\text {FADT }}$ compile the dependencies on $\left(e, T, d_{0}\right)$ and can be viewed as viscosities for volumetric and deviatoric viscous deformations. In fact, when void ratio goes to zero, only the deviatoric behaviour remains and the viscous deformation is completely analogous to a Newtonian fluid. It should be noticed that $\mathrm{d} \varepsilon_{\mathrm{v}} / \mathrm{d} t=0$ for $e=0$ due to the proposed form of chemical potential at pores (7b).

An important result is that for this mechanism a linear dependence of deformation rate on stresses is found. This statement is not completely true because strain rates are, in fact, a function of void ratio. So, if void ratio could be considered constant over a small range of deformations, then (7a) and (7b) would express a linear viscoelastic behaviour. If void ratio changes, (7a) and (7b) are non-linear and a steady-state behaviour does not exist.

Another point that deserves further comments is the presence of brine in pores. It has been assumed that the medium is fully saturated with brine. However, experiments from Reference [17] show that full saturation is not a necessary condition for this mechanism to operate.

Without loss of generality, for unsaturated conditions effective stresses $\left(\sigma_{i}^{\prime}=\sigma_{i}-P_{\ell}\right)$ should change into another definition $\left(\sigma_{i}^{\prime}=\sigma_{i}-P_{\mathrm{g}}\right)$ in $(5 \mathrm{a})$ and $(5 \mathrm{~b})$ where $P_{\mathrm{g}}$ is the pressure of the gas phase, which is used in soil mechanics [26]. The term $P_{\ell} V_{\mathrm{m}}$, does not change in (5a) and (5b) because both the contact and the pore are assumed to be in contact with the liquid phase.

On the other hand, experimental results have shown (see Figure 11) that a strain rate reduction takes place under unsaturated conditions, specially at low saturation levels. This can be explained by a reduction of brine film thickness $(a)$ due to suction forces and/or an increase of tortuosity (reduction of $\tau$ ). To take into account this effect, an empirically derived dependence on the square root of degree of saturation $\left(S_{\ell}\right)$ has been adopted. Backfills of porous salts are likely to have small quantities of brine which may be sufficient for the FADT mechanism to be active. Figure 11 shows the strain rate reduction that takes place as the medium becomes unsaturated. 


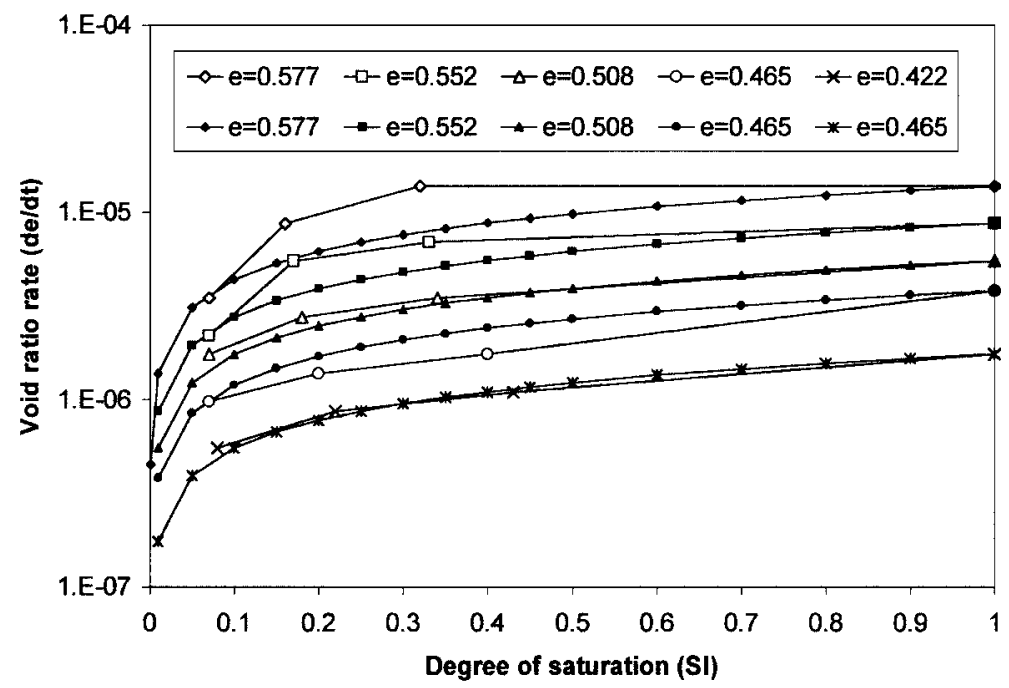

Figure 11. Void ratio rate of tests at $4.2 \mathrm{MPa}, 22^{\circ} \mathrm{C}$, grainsize $275 \mu \mathrm{m}$ and constant brine content. Points with the same void ratio are connected. Experimental data from Spiers et al. [17]: open symbols; function of square root of degree of saturation plotted in closed symbols.

2.3.5. Generalization of the constitutive relationships for FADT. The final equations for FADT mechanism express strain rate as a function of several variables (Equation (6)). They are: stresses, void ratio, grain size, temperature and medium properties. The linear dependence on stresses allows to postulate a viscoelastic form. Equations (7a) and (7b) show that the dependence of strain rates on stresses can be separated from the other variables leading to two laws apparently independent for volumetric and deviatoric components of deformation. If one knows these two components, it is straightforward to generalize the model with a viscoelastic law (here including also the correction for unsaturated conditions):

$$
\frac{\mathrm{d} \varepsilon_{i j}^{\mathrm{FADT}}}{\mathrm{d} t}=\frac{1}{2 \eta_{\mathrm{FADT}}^{\mathrm{d}}}\left(\sigma_{i j}^{\prime}-p^{\prime} \delta_{i j}\right)+\frac{1}{3 \eta_{\mathrm{FADT}}^{\mathrm{v}}} p^{\prime} \delta_{i j}
$$

where $\sigma_{i j}^{\prime}$ is the effective stress tensor (defined as $\sigma_{i j}^{\prime}=\sigma_{i j}^{\prime}-\delta_{i j} P_{\mathrm{f}}$ ), $P_{\mathrm{f}}$ is the fluid pressure (gas if unsaturated and liquid if saturated $), p^{\prime}$ is the mean effective stress $\left(p^{\prime}=\left(\sigma_{11}^{\prime}+\sigma_{22}^{\prime}+\sigma_{33}^{\prime}\right) / 3\right), \delta_{i j}$ is the Kronecker delta, and the volumetric and deviatoric viscosities are defined from (7a) and (7b), respectively:

$$
\begin{gathered}
\frac{1}{\eta_{\mathrm{FADT}}^{\mathrm{v}}}=\frac{16 B(T) \sqrt{S_{\ell}}}{d_{0}^{3}} g_{\mathrm{FADT}}^{\mathrm{v}}(e)=C\left(T, S_{\ell}, d_{0}\right) g_{\mathrm{FADT}}^{\mathrm{v}}(e) \\
\frac{1}{2 \eta_{\mathrm{FADT}}^{\mathrm{d}}}=\frac{16 B(T) \sqrt{S_{\ell}}}{d_{0}^{3}} g_{\mathrm{FADT}}^{\mathrm{d}}(e)=C\left(T, S_{\ell}, d_{0}\right) g_{\mathrm{FADT}}^{\mathrm{d}}(e) \\
g_{\mathrm{FADT}}^{\mathrm{v}}(e)=\frac{3 g^{2} e^{3 / 2}}{(1+e)}, \quad g_{\mathrm{FADT}}^{\mathrm{d}}(e)=\frac{g^{2}}{(1+e)}
\end{gathered}
$$


The function $C\left(T, S_{\ell}, d_{0}\right)$ includes the information related essentially to grain size, brine content and temperature and the function $g(e)$ has the form

$$
g=\frac{1}{(1-f)^{2}}=\frac{d^{2}}{x^{2}}, \quad f=\sqrt{\frac{2 e}{3\left(1-e^{3 / 2}\right)(1+e)}}=\frac{s \sqrt{2}}{d}
$$

i.e. $g$ and $f$ are geometrical functions related to $d / x$ and $s / d$. Associated with volumetric and deviatoric behaviour, the auxiliary functions $g_{\text {FADT }}^{\mathrm{v}}$ and $g_{\text {FADT }}^{\mathrm{d}}$ contain the dependence on void ratio. These functions are plotted in Figure 12.

The functions $g_{\mathrm{FADT}}^{\mathrm{v}}$ and $g_{\mathrm{FADT}}^{\mathrm{d}}, g$ and $f$ are geometry dependent. In principle, change in other grain shapes would modify these functions, while the other dependences would remain unchanged. The shape obtained with the geometry used in this paper, as will be shown later, seems to be sufficiently adequate.

Finally, it should be emphasized that Equation (8) shows a linear dependency with respect to stress but, as mentioned, it is in fact a non-linear relationship because void ratio, which appears in the viscous coefficients, depends on strains. In this context, the non-linear law given by Equation (8) is still referred to as viscoelasticity due to its mathematical form. However, this would be strictly true if the viscosity coefficients were constant with void ratio.

\subsection{Dislocation creep mechanism}

The second mechanism included in the model is called DC (dislocation creep strain). With this name several strain mechanisms related to dislocation theory are grouped. The most important one is dislocation climb, but glide of dislocations can also take place. The main reason for this grouping is that these mechanisms can be represented by power-law terms, i.e. the same mathematical representation corresponds to various possible strain mechanisms. A very comprehensive discussion of the relevance of the different mechanisms of crystalline deformation in salt rock can be found in Reference [9].

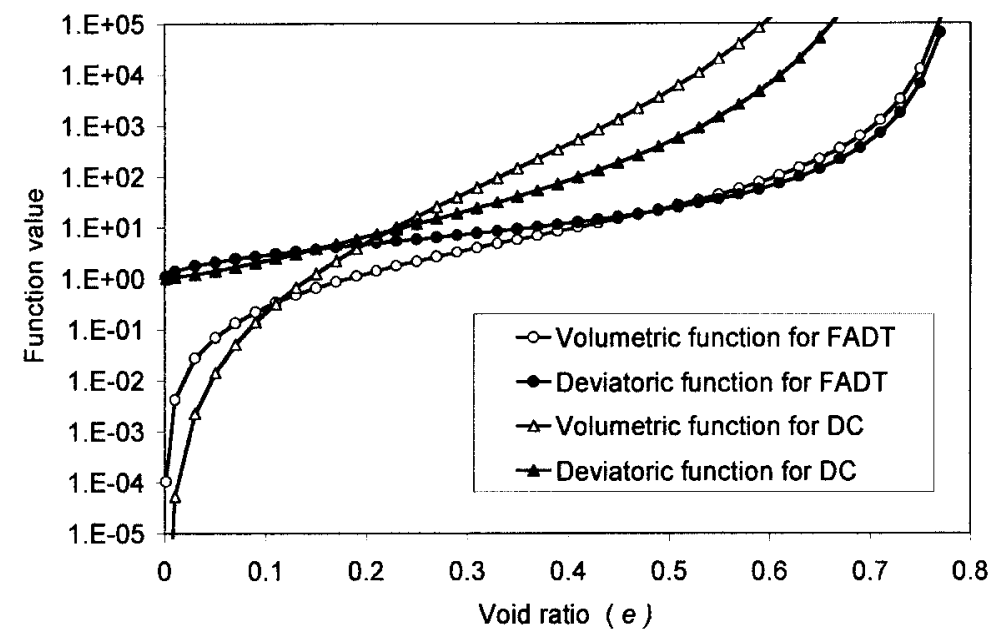

Figure 12. Auxiliary functions $g_{\mathrm{FADT}}^{\mathrm{v}}, g_{\mathrm{FADT}}^{\mathrm{d}}, g_{\mathrm{DC}}^{\mathrm{v}}$ and $g_{\mathrm{DC}}^{\mathrm{d}}$ (Equations (10) and (18)). 
As explained (Section 1.2) creep of rock salts is governed by a power law as a first approximation. This law, can be written in different ways, the most common is

$$
\frac{\mathrm{d} \varepsilon_{i j}}{\mathrm{~d} t}=A q^{n} \frac{\partial q}{\partial \sigma_{i j}}
$$

in the generalized case $\left(q=\left(3 J_{2}\right)^{0.5}\right.$ deviatoric stress, $J_{2}$ : second invariant of the deviatoric part of the stress tensor). $A(T)$ is a temperature-dependent parameter, and $n$ is the power of the creep law. It is important to notice that this law does not allow volumetric creep deformation. This power law will be combined with the selected idealized geometry to obtain a law for porous aggregates.

Yet, another form of the power law can be obtained if the generalized one is particularized for the principal directions:

$$
\frac{\mathrm{d} \varepsilon_{i}}{\mathrm{~d} t}=\frac{3}{2} A q^{n-1}\left(\sigma_{i}-p\right)
$$

It does not matter if effective or total stress is used here because deviatoric stresses do not depend on pore pressure and this law does not contain volumetric contribution. Therefore, for porous materials, net stresses $\left(\sigma_{i j}^{\prime}=\sigma_{i j}^{\prime}-P_{f} \delta_{i j}\right)$ will be considered in what follows.

From the basic mechanism equations and the proposed geometry a macroscopic law is sought. However, it is not possible to obtain analytical expressions without making further assumptions. The following hypotheses will be considered:

- A simple stress distribution inside the grain.

- The grains deform, i.e. change their shape without volume change.

- Creep power law is valid to calculate the deformation of the grain.

A direct consequence of these assumptions is that volumetric deformation will be produced by the deformation of the grains but not by their reorganization. The macroscopic equations of the model will be built in three steps. These are: adoption of a stress distribution in a grain, calculation of strain rates along the principal directions and generalization of the model to a tensorial form.

2.4.1. Stress distribution in a grain. Figure 13 shows the cross-section of the grain and different zones are distinguished, $o, i$ and $j$ ( $k$ would appear in another cross-section). It is assumed that in zone $o$ the stress state is equal to the macroscopic one. So the core of the grains has similar stress state as externally applied. In zone $i$, near the contacts, the stress state is modified by the influence of stress concentration. This is expressed by $\left(\sigma_{i}^{\prime}\right)_{\mathrm{c}},\left(\sigma_{j}^{\prime}\right)_{\mathrm{c}}$ and $\left(\sigma_{k}^{\prime}\right)_{\mathrm{c}}$, i.e. the corresponding principal effective stress is concentrated while the other remain equal to the macroscopic ones. Concentration is obtained as $\left(\sigma_{i}^{\prime}\right)_{\mathrm{c}}=\left(\sigma_{i}^{\prime}\right) d^{2} / x^{2}$.

The assumption of using averaged stresses in the various zones considered is indicated in Figure 13 as piecewise. The elastic stress distribution in a grain was also calculated by finite element computations at grain level [27]. The stress inside the grain could be considered a function of the distance to the contact, but using piecewise values strongly simplifies the development of the model equations. Other possibilities can be considered in future refinements of the model. 


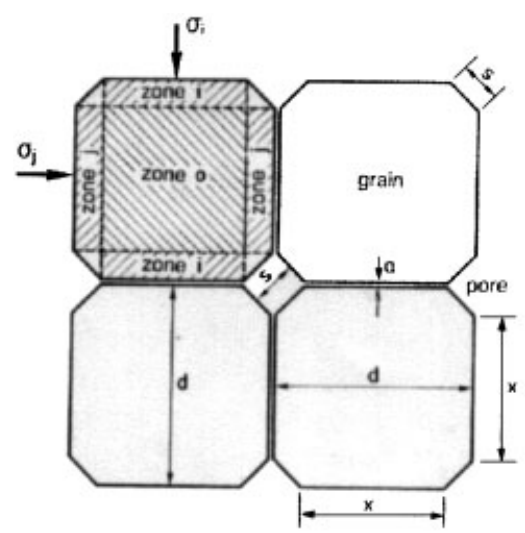

(a)

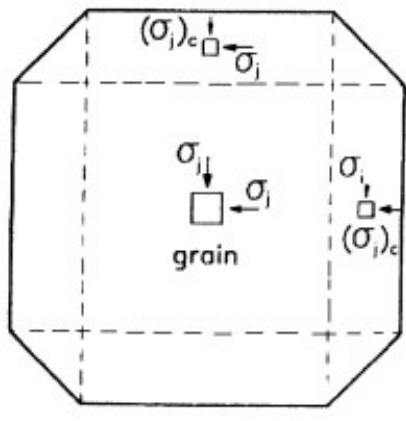

(b)

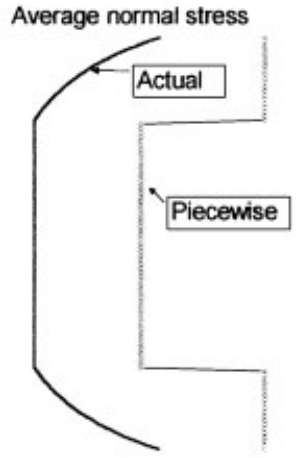

(c)

Figure 13. Idealized grain section: (a) characteristic sizes in the geometry; (b) central zone and contact zone in the grain section; and (c) average normal stress (vertical stress in this case) along horizontal sections (maximum stress on the contact and minimum stress in the grain core).

2.4.2. Strain rates along principal directions. The assumed stress distribution inside a grain is used to compute the strain rate along the principal direction $i$. As the size of the grain $d$ changes with time, strain rate along any principal direction $i$ can be estimated as $((1 / d) \mathrm{d} d / \mathrm{d} t)_{i}$, where $\mathrm{d} d / \mathrm{d} t$ is computed using (13) in the different grain zones described above:

$$
\frac{\mathrm{d} \varepsilon_{i}}{\mathrm{~d} t}=\left(\frac{1}{d} \frac{\mathrm{d} d}{\mathrm{~d} t}\right)_{i} \cong \frac{3}{2} A q_{i}^{n-1}\left(\left(\sigma_{i}^{\prime}\right)_{\mathrm{c}}-p_{i}^{\prime}\right) \frac{\sqrt{2} s}{d}+\frac{3}{2} A q^{n-1}\left(\sigma_{i}^{\prime}-p^{\prime}\right) \frac{x}{d}
$$

where $p_{i}^{\prime}$ and $q_{i}$ are mean net stress and deviatoric stress in zone $i$ (Figure 13), i.e. computed with $\left(\sigma_{i}^{\prime}\right)_{\mathrm{c}},\left(\sigma_{j}^{\prime}\right)_{\mathrm{c}}$ and $\left(\sigma_{k}^{\prime}\right)_{\mathrm{c}}$; and, $p^{\prime}$ and $q$ are mean net stress and deviatoric stress in zone $o$, i.e. computed with $\left(\sigma_{i}^{\prime}\right),\left(\sigma_{j}^{\prime}\right)$ and $\left(\sigma_{k}^{\prime}\right)$. In this equation, the first term corresponds to the strain of the area near contacts and the second term corresponds to the strain in the core of the grain. As porosity tends to zero, the first term vanishes, and the original law for rock salt is obtained. The volumetric strain law for DC could be obtained simply by addition of strain rates along the three principal directions. However, a more simple relationship is obtained if the stress state is considered isotropic, i.e. $\left(\sigma_{1}^{\prime}\right)=\left(\sigma_{2}^{\prime}\right)=\left(\sigma_{3}^{\prime}\right)$ :

$$
\begin{aligned}
\frac{\mathrm{d} \varepsilon_{\mathrm{v}}}{\mathrm{d} t} & =\frac{\mathrm{d} \varepsilon_{1}}{\mathrm{~d} t}+\frac{\mathrm{d} \varepsilon_{2}}{\mathrm{~d} t}+\frac{\mathrm{d} \varepsilon_{3}}{\mathrm{~d} t}=3 A\left(\left(p^{\prime}\right)_{\mathrm{c}}-p^{\prime}\right)^{n} \frac{\sqrt{2} s}{d} \\
& =3 A\left(\frac{(1+e)}{\left(\sqrt{1+e}-\sqrt{2 e / \lambda_{\mathrm{v}}}\right)^{2}}-1\right)^{n} \frac{\sqrt{2 e / \lambda_{\mathrm{v}}}}{\sqrt{1+e}}\left(p^{\prime}\right)^{n}=\frac{1}{\eta_{\mathrm{v}}^{\mathrm{DC}}(e, T)}\left(p^{\prime}\right)^{n}
\end{aligned}
$$

As an opposite state to isotropic, pure shear stress state is considered, i.e. $\left(\sigma_{3}^{\prime}\right)=-\left(\sigma_{1}^{\prime}\right)$ and $\left(\sigma_{2}^{\prime}\right)=0$ :

$$
\frac{\mathrm{d} \varepsilon_{1}}{\mathrm{~d} t}=\frac{3}{2} A(T)\left(\left(\sqrt{1+g+g^{2}}\right)^{n-1}\left(\frac{2 g+1}{3}\right) f+(\sqrt{3})^{n-1} \frac{1}{\sqrt{g}}\right) \sigma_{1}^{\prime n}
$$


The reason for these two particularizations (isotropic and pure shear) is to obtain simple relationships for volumetric strain rate and deviatoric strain rate. If a general stress state was assumed without any restrictions, these forms would be more complex due to the non-linear (power) dependence of strain rate on stress for this mechanism. From (15) and (16) the following viscosities for volumetric and deviatoric creep can be obtained:

$$
\begin{aligned}
& \frac{1}{\eta_{\mathrm{DC}}^{\mathrm{v}}}=A(T) g_{\mathrm{DC}}^{\mathrm{v}}(e) \\
& \frac{1}{\eta_{\mathrm{DC}}^{\mathrm{d}}}=A(T) g_{\mathrm{DC}}^{\mathrm{d}}(e)
\end{aligned}
$$

where

$$
\begin{aligned}
& g_{\mathrm{DC}}^{\mathrm{v}}(e)=3(g-1)^{n} f \\
& g_{\mathrm{DC}}^{\mathrm{d}}(e)=\left(\sqrt{\frac{1+g+g^{2}}{3}}\right)^{n-1}\left(\frac{2 g+1}{3}\right) f+\frac{1}{\sqrt{g}}
\end{aligned}
$$

where $g$ and $f$ have already been defined in (11), $A(T)$ and $n$ come directly from the creep power law for rock salt, i.e. the non-porous material. The functions $g_{\mathrm{DC}}^{\mathrm{v}}(e)$ and $g_{\mathrm{DC}}^{\mathrm{d}}(e)$ depend on the idealized geometry and in turn on void ratio. However, because of the originally non-linear power law, a dependence on the power $n$ remains in these functions. Since this parameter $(n)$ does not change very much in saline materials, this power in the geometrical function does not represent a practical difficulty. A value of $n=5$ has been used to calculate these functions (Figure 12) which is a theoretical value for dislocation climb plus glide [28].

The geometrical functions for FADT and for DC mechanisms show similar behaviour, but for $\mathrm{DC}$ a much higher increase is observed as void ratio increases.

2.4.3. Generalization of the model for DC. Equation (14) has been developed on the basis of the idealized geometry adopted. However, it lacks generality because it has been written for strain rates along principal directions. While for FADT, linear dependences could be exploited in order to obtain a viscoelastic generalization, for DC this is not possible. One way to generalize (14) is to use a flow rule. In this procedure, (15) and(16) will be very useful to identify how this generalized form should be. The following general form, based on viscoplasticity theories for geological materials [29], is proposed:

$$
\frac{\mathrm{d} \varepsilon_{i j}^{\mathrm{DC}}}{\mathrm{d} t}=\frac{1}{\eta} \Phi(F) \frac{\partial G}{\partial \sigma_{i j}^{\prime}}
$$

In this equation, a viscosity parameter is included, $G$ is a flow rule, $F$ is a stress function and $\Phi$ is a scalar function.

In Equation (19), $G$ and $F$ should be taken as functions of stress invariants. The following form is proposed here:

$$
\begin{aligned}
F & =G=\sqrt{q^{2}+\left(\frac{p}{\alpha_{\mathrm{p}}}\right)^{2}} \\
\Phi(F) & =F^{n}
\end{aligned}
$$


where $n$ is the power of the rock creep law and $\alpha_{\mathrm{p}}$ is a material parameter. Figure 14 shows the shape of the flow rule in the $\left(p^{\prime}, q\right)$ plane depending on $F$ and $\alpha_{\mathrm{p}}$ values.

Since $\Phi(F)$ is always positive, no threshold is considered in this law. This is consistent because saline rocks develop viscous deformations under any stress level. In order to exploit the theoretically derived equations ((14)-(16)) this generalization of the model is complemented with definitions for $\eta$ and $\alpha_{\mathrm{p}}$. By comparison of (15) and (16) with the results obtained using (19) and (20) under the same stress state (i.e. isotropic and pure shear) it is obtained that

$$
\eta=\eta_{\mathrm{DC}}^{\mathrm{d}}, \quad \alpha_{\mathrm{p}}=\left(\frac{\eta_{\mathrm{DC}}^{\mathrm{v}}}{\eta_{\mathrm{DC}}^{\mathrm{d}}}\right)^{1 /(n+1)}
$$

in other words, using (19)-(21) produces exactly the same results as would be obtained using (14) for the cases of isotropic (i.e Equation (15)) and pure shear stress state (i.e. Equation (16)), respectively.

When void ratio vanishes, $\alpha_{\mathrm{p}}$ tends to infinity and the mathematical expression of $G$ (Equation (20)) reduces to the von Mises case. In this way the creep law for rock salt with von Mises flow rule is recovered. Therefore, $\alpha_{\mathrm{p}}$ can be seen as a hardening parameter for volumetric creep behaviour. With the flow rule adopted, the model does not allow any dilatancy behaviour, and it is expected to be adequate only for high confinement situations. This limitation can be overcome in the future if other forms for the flow rule and stress function $(G$ and $F$ ) are explored. This would require to look carefully at the behaviour under dilatant conditions of porous aggregates.

\subsection{Creep model equation}

The physical description of the model for creep presented in this paper indicates that two independent deformation mechanisms contribute to the total strain rate. FADT is intercrystal-

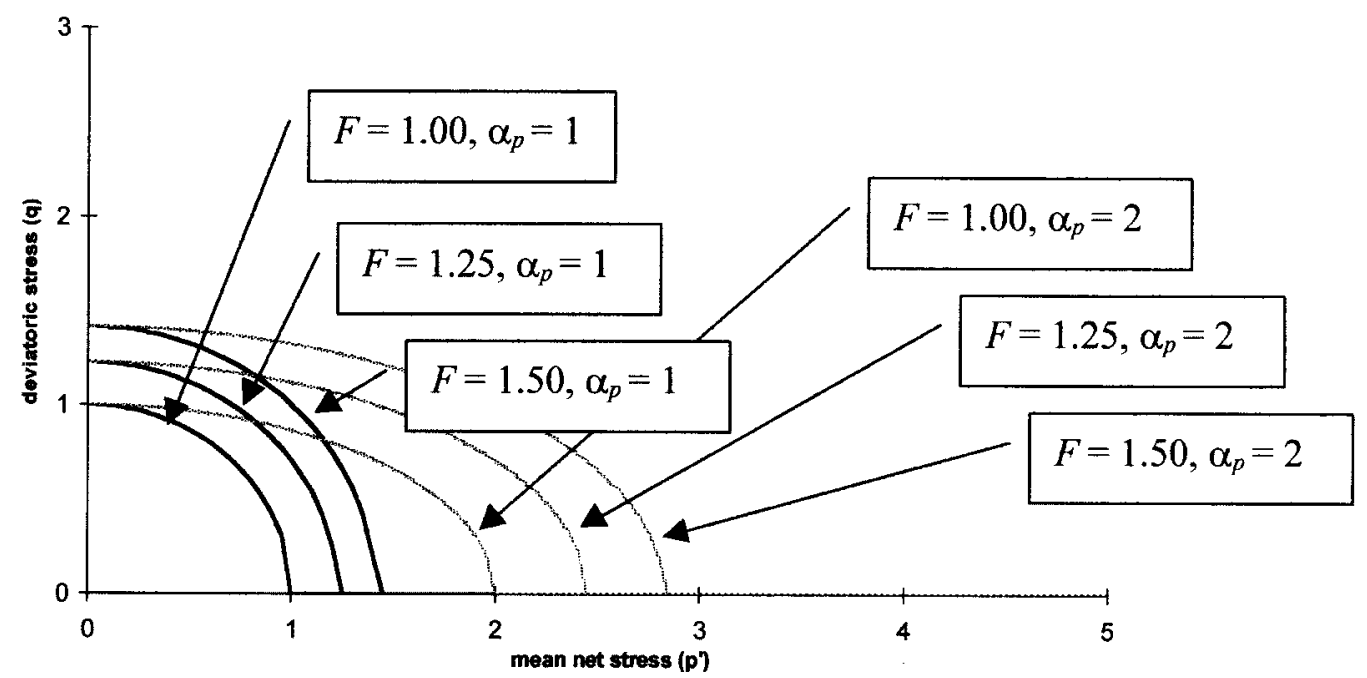

Figure 14. Stress function and flow rule $F=G$ used for the viscoplastic generalization (DC mechanism). 
line while DC is intracrystalline. Hence, it is reasonable to combine them in a single additive way. Therefore, creep strain rate can be written as the sum of Equations (8) and (19), that is

$$
\frac{\mathrm{d} \varepsilon_{i j}^{\mathrm{c}}}{\mathrm{d} t}=\frac{\mathrm{d} \varepsilon_{i j}^{\mathrm{FADT}}}{\mathrm{d} t}+\frac{\mathrm{d} \varepsilon_{i j}^{\mathrm{DC}}}{\mathrm{d} t}=\frac{1}{2 \eta_{\mathrm{FADT}}^{\mathrm{d}}}\left(\sigma_{i j}^{\prime}-p^{\prime} \delta_{i j}\right)+\frac{1}{3 \eta_{\mathrm{FADT}}^{\mathrm{v}}} p^{\prime} \delta_{i j}+\frac{1}{\eta_{\mathrm{DC}}^{\mathrm{d}}} \Phi(F) \frac{\partial G}{\partial \sigma_{i j}^{\prime}}
$$

The set of variables temperature, stress level, grain size and brine content can give a predominance of one mechanism over the other. For instance, if the medium is absolutely dry, or, it has a very large grain size, DC will predominate over FADT. On the contrary, if grain size is small (say of the order of hundreds of microns) and brine is present, FADT will predominate, specially at low stresses.

It must be noticed that accumulation of volumetric deformations will change the void ratio, thus changing viscosities. From this point of view, the mechanisms are not independent. For instance, DC will take place at the same strain rate if stress level, void ratio and temperature are the same in two samples, one completely dry and the other fully saturated. Of course, after some time, the saturated sample will have reduced void ratio more than the dry sample. At that point, DC strain rate will not be the same in the two samples because void ratio will not be the same.

\subsection{Main contributions of this model}

The main contributions of the model presented in this paper are

- A new idealized geometry has been adopted valid for void ratios from $e=0$ to 0.78 (the geometry it is used for both FADT and DC).

- Estimation of chemical potential gradients from contacts to pores in a way that produces viscous incompressibility at full compaction (for FADT).

- Strain rates for DC are estimated from grain deformation assuming a stress distribution inside the grains (from DC). Viscous incompressibility is achieved at full compaction.

- The theoretically derived equations have been generalized to a tensorial form (for both FADT and DC).

- Combination of the two mechanisms under the assumption that they are independent from the physical point of view. Degree of saturation weights the FADT mechanism. Void ratio couples both mechanisms in porous aggregates because viscosities for both mechanisms depend on it.

\section{MODEL PREDICTIONS USING THE CREEP EQUATIONS}

The predictions of the model have been compared with isotropic and oedometric creep test results reported in the literature under dry and saturated conditions. The same values for $B(T)$ and $A(T)$ have been used for all comparisons regardless of the other variables (e.g. stress, grain size). In fact $B(T)$ and $A(T)$ correspond to salt-rock properties, therefore they must depend, in principle, only on the nature of the medium, in this case halite.

In the model predictions included in this paper we have used the following values: DC:

$$
A(T)=5 \times 10^{-6} \exp \left(\frac{-59650}{R T}\right) \mathrm{s}^{-1} \mathrm{MPa}^{-n}, \quad n=5.375
$$


FADT creep:

$$
B(T)=\frac{6 \times 10^{-13}}{R T} \exp \left(\frac{-24530}{R T}\right) \mathrm{s}^{-1} \mathrm{MPa}^{-1} \mathrm{~m}^{3}
$$

The two parameters have different units because each mechanism of deformation exhibits different dependences. For DC the adopted values correspond to typical values for rock salt (from Asse mine) deviatoric creep deformation [30]. An experimental value of $n=5.375$ was obtained instead of the theoretical $n=5$ value. For FADT, the constant inside the exponential has been taken from Spiers et al. [18] while the pre-exponential constant has been determined to obtain a good prediction of the experimental test (see below) represented in Figure 15. Finally, void ratio rate can be obtained as $\mathrm{d} e / \mathrm{d} t=(1+e) \mathrm{d} \varepsilon_{\mathrm{v}} / \mathrm{d} t$.
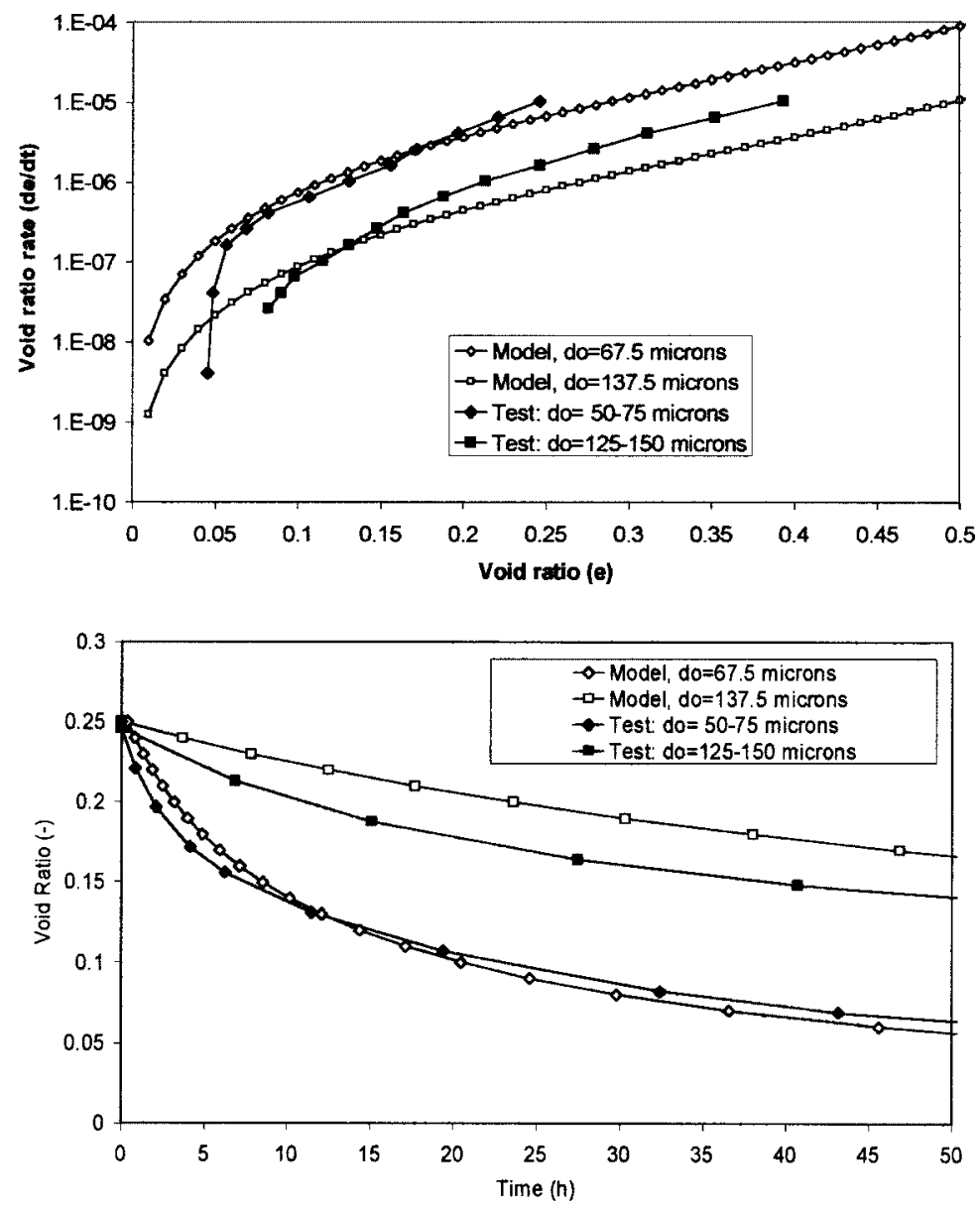

Figure 15. Comparison of model predictions (creep FADT mechanism) with experimental results from creep tests on saturated crushed salt (data from Reference [24]). Time evolution is represented only from $e=0.25$. 
Figures 15 and 16 show comparisons with test results obtained in the literature. Figure 15 is a clear example of deformation controlled by FADT mechanism because of the small grain size of the material used (50-75 and 125-150 $\mu \mathrm{m})$, the low stress level and the presence of brine. On the contrary, Figure 16 shows two tests carried out under relatively dry conditions, higher stresses and, in one of them, higher temperature. Therefore, in this case, the predominant strain mechanism is DC. A quite good prediction of strain rates is obtained in both cases.
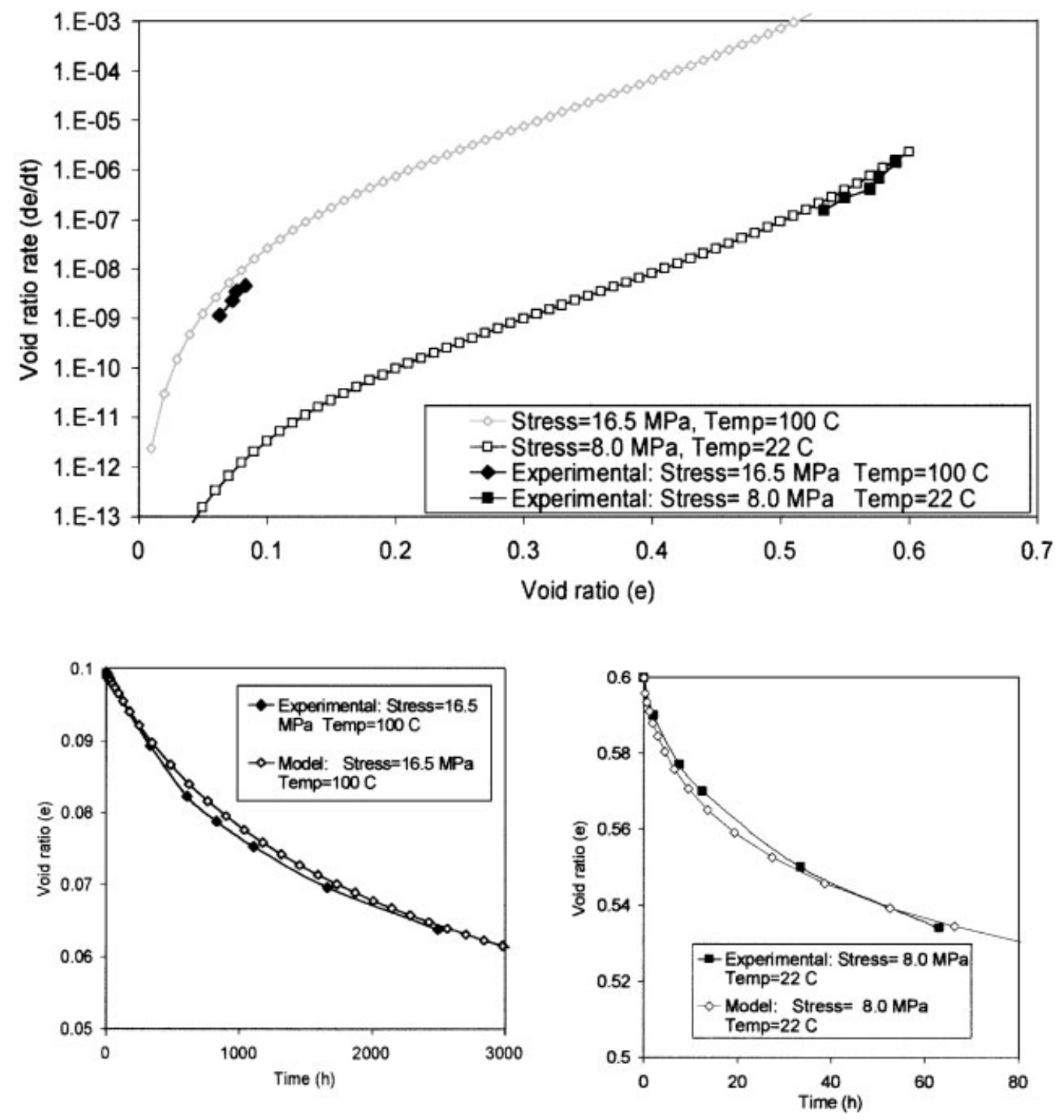

Figure 16. Comparison of model predictions (DC mechanism) with experimental results from creep tests on dry crushed salt. One test is performed with a stress of $16.5 \mathrm{MPa}$ and at $100^{\circ} \mathrm{C}$ while the other is performed with a stress of $8.0 \mathrm{MPa}$ and at $22^{\circ} \mathrm{C}$ (data from Reference [17]). 
The results of an oedometric laboratory test carried out in our laboratory have also been used to compare the predictions of the model. The test was carried out at $25^{\circ} \mathrm{C}$ and under brinesaturated conditions. The salt powder was obtained at the Suria mine (Spain) and the sample prepared with a controlled grain size of $0.520 \mathrm{~mm}$. Four steps of stress were applied to the sample, these are: $0.6,3.7,6.0$ and $30 \mathrm{MPa}$. The high load for the last stage was mainly used in order to finish the test in a shorter time. Such high-stress state can only be achieved in a repository backfill at low void ratios, i.e. after strong compaction.

Figure 17 shows creep strain rates as a function of void. This representation is very useful because it allows the estimation of the creep parameters. In this test, the contribution of both deformation mechanisms was considered because of the high stress used during the last loading step (30 MPa). One way to see the mechanism that is predominating is to compare the shape of the curves $\mathrm{d} e / \mathrm{d} t$ vs $e$ with the shape of the functions $g_{\mathrm{FADT}}^{\mathrm{v}}$, and $g_{\mathrm{DC}}^{\mathrm{v}}$ plotted in Figure 12. DC dominates for the highest stress level $(30 \mathrm{MPa})$ in almost all the range of void ratios $(e>0.1)$. For stress levels of 3.7 and $6 \mathrm{MPa}, \mathrm{DC}$ is dominant only if void ratio is very high $(e>0.6)$. FADT dominates in the other cases. The change of the regime defined by one or other mechanism takes place in a continuous way, so that, there is a transition zone in which both mechanisms give similar contribution.

The creep strain rates do not predict very well the transient period immediately after the stress increment. This should be expected because the model does not include a component for the quasi-instantaneous deformations that occur immediately after loading due to particle rearrangement. This is discussed in Section 4. Here, only an elastic contribution has been considered with a Young's modulus of $2000 \mathrm{MPa}$ and a Poisson's modulus of 0.30 .

A deviation of strain rates is obtained for low void ratios. The observed strain rates are smaller than the ones predicted with the model. It should be pointed out that the model assumes perfect connectivity of pores until they close completely. In reality, brine flow may be restricted by the low porosity of the medium causing a smaller rate of creep than the one derived from the model. At low void ratio, therefore, permeability may control deformation. A similar difference can be observed in the modelling of tests reported by Schutjens [24] (Figure 15). A coupled microstructural analysis of the experiment itself would, in principle, permit the modelling of this phenomenon.

In spite of the apparent complexity of the model, the number of free parameters is, in fact, highly reduced and most equations are derived from the idealized geometry adopted. In fact, most parameters correspond to either well-established physical constants or to rock salt parameters. An additional feature is the fact that at low void ratio only the DC creep component operates and a smooth transition to rock salt behaviour is obtained. In this way the full transformation from granular material to soft rock is accounted for. Although the number of free parameters is small, the overall model is able to reproduce observed behaviour quite satisfactorily.

\section{COMBINATION OF CREEP EQUATIONS AND A VISCOPLASTIC LAW}

Originally, the creep equations were combined with an elastic part. This was not enough for representing mechanisms of deformation that may take place related to grain reorganization and sliding. These mechanisms are essentially irreversible because changes in the structure take place and the original arrangement cannot be recovered. These mechanisms are typical mechanisms of soil deformations. In fact, if the tests in salt are performed at constant strain rate instead of at constant stress, the results can hardly be modelled with the creep equations combined with elasticity. 

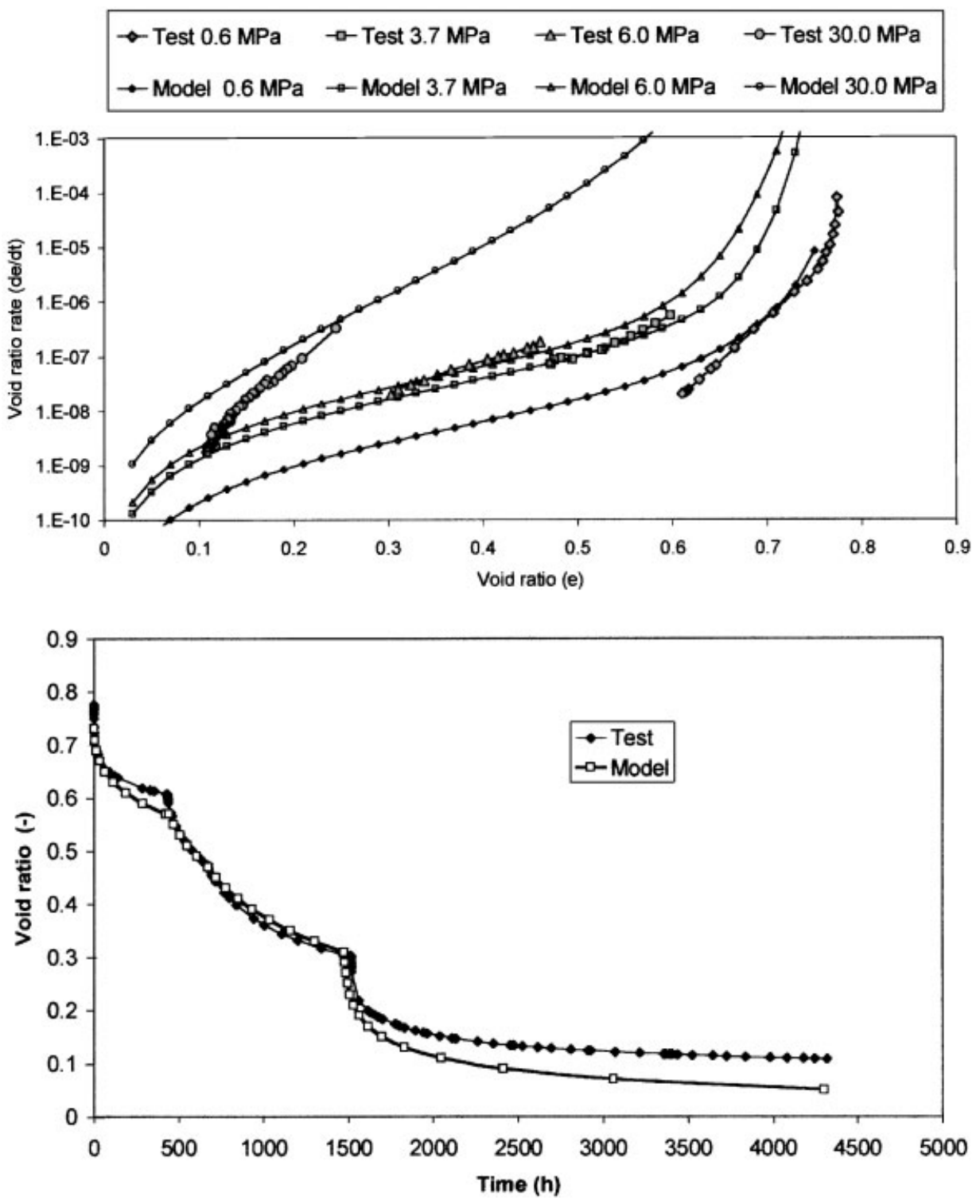

Figure 17. Comparison of oedometer test results with full model predictions; tests on Suria crushed salt.

In the context of a European Community project named BAMBUS, experimental data were available on variable-stress conditions. For example, Figure 18 shows experimental results of crushed salt compaction at different velocities. Void ratio (or porosity) versus stress (or logstress) is a typical representation in soil mechanics. From Figure 18 it can be seen that the faster the loading, the smaller the deformation indicating that there is an important effect of loading velocity. A creep deformation that develops during loading is an explanation of the difference. 

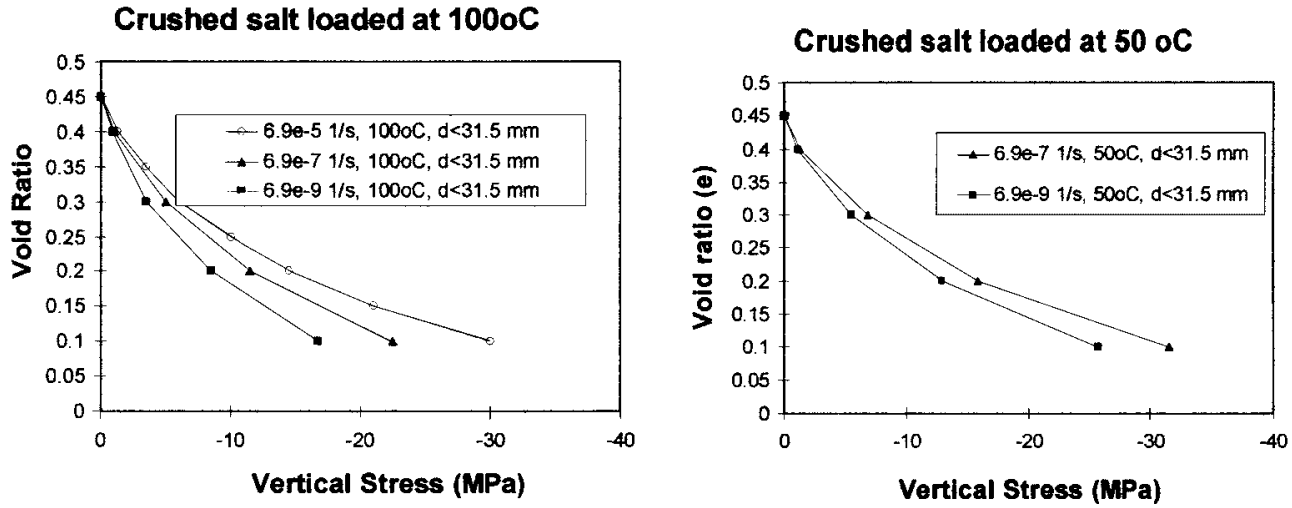

Figure 18. Results of constant strain rate compression tests.

In order to model this behaviour, it is necessary to incorporate another component on the model. Figure 19 shows an ideal representation of the combined processes of deformation that can explain the mechanical behaviour of crushed salt. A loose aggregate will respond as a sand, specially if loading takes place fast. Modelling of such loose material can be made with a critical state model, which is based on a yield surface. This yield surface, corresponding to an unbonded granular material, will expand due to densification and bond creation. Expansion implies increase of $p_{0}$ and decrease of $p_{t}$. Bond development tends to be more closely associated with an increase of cohesion (controlled by parameter $p_{t}$ ), whereas compaction increases preferentially the value of $p_{0}$. The yield surface at a given state defines an internal domain of elastic plus creep behaviour. As mentioned above, creep is not lower bounded by any threshold. Even if the stress level falls inside a given yield surface, densification is still progressing and the yield surface expanding. Under loading, elastic and creep deformations are combined with viscoplastic deformations as the yield surface is reached by the stress point.

Based on these ideas, a viscoplastic law is chosen in order to make the model more comprehensive. The basic viscoplastic equations are as follows:

$$
\begin{aligned}
\frac{\mathrm{d} \varepsilon_{i j}^{\mathrm{VP}}}{\mathrm{d} t} & =\frac{1}{\eta_{\mathrm{VP}}^{\mathrm{d}}}\left\langle\Phi\left(F^{\mathrm{VP}}\right)\right\rangle \frac{\partial F^{\mathrm{VP}}}{\partial \sigma_{i j}^{\prime}} \\
F^{\mathrm{VP}} & =\sqrt{q^{2}+M^{2}\left(p^{\prime}+p_{t}\right)\left(p^{\prime}-p_{0}\right)} \\
p_{0} & =a\left(\varepsilon_{\mathrm{v}}^{\text {inelastic }}\right)^{\mathrm{m}}, \quad p_{t}=b\left(\varepsilon_{\mathrm{v}}^{\text {inelastic }}\right)^{\mathrm{p}} \\
\Phi\left(F^{\mathrm{VP}}\right) & =\left(F^{\mathrm{VP}}\right)^{n} \\
\frac{1}{\eta_{\mathrm{VP}}^{\mathrm{d}}} & =A_{\mathrm{VP}} \exp \left(\frac{-Q_{\mathrm{VP}}}{R T}\right) g^{\mathrm{d}}\left(e^{\prime}\right) \\
e^{\prime} & =e+\frac{e^{3}}{e_{0}^{3}}\left(e_{\max }-e_{0}\right)
\end{aligned}
$$




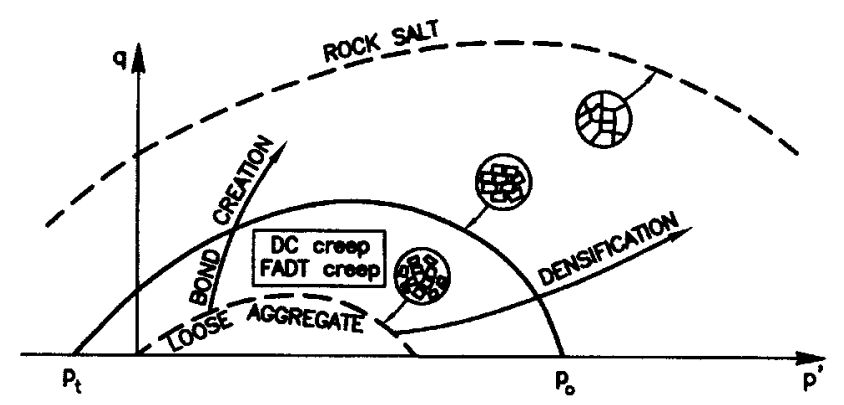

Figure 19. Mechanisms of deformation in crushed salt.

where $F$ is the viscoplastic yield function, $p_{0}$ and $p_{t}$ are the hardening parameters, $\sigma^{\prime}$ is the effective stress (soil mechanics) and $\varepsilon$ strain. An important feature of the model is that the hardening is, in most situations, driven by the inelastic deformations arising from the creep mechanisms. The combination of the viscoplastic component of the model together with the creep DC component was considered for modelling crushed salt in the context of the BAMBUS project [31]. The following set of parameters was obtained for the model:

$$
\begin{array}{ll} 
& n=3, \quad m=4, \quad a=3400, \\
\text { viscoplastic component }: & Q_{\mathrm{VP}}=54000 \mathrm{~J} / \mathrm{mol}, \quad A_{\mathrm{VP}}=0.005(1 / \mathrm{s}), \quad M=1 \\
& e_{\max }=0.75, \quad e_{0}=0.45
\end{array}
$$$$
\text { creep component : } n=5, \quad Q_{\mathrm{DC}}=54000 \mathrm{~J} / \mathrm{mol}, \quad A_{\mathrm{DC}}=5.810^{-6}(1 / \mathrm{s})
$$

This set of parameters was calibrated and the strain rates are represented in Figure 20 compared with the measured strain rates at 62 points corresponding to several triaxial tests carried out by Korthaus at FZK in Germany [32]. Manual minimization of the average error for all points was performed. The calibration over these 62 values is considered comprehensive since porosity ranges from 0.05 to 0.25 , mean stresses from 2 to $12 \mathrm{MPa}$ and temperatures from 25 to $150^{\circ} \mathrm{C}$.

\section{CONCLUSIONS}

In this paper a complete constitutive model for crushed salt is presented. Crushed salt evolves from high-porosity conditions to a nearly rock-like material by volumetric deformation. Different stress, temperature and humidity conditions may lead to different mechanisms of compaction. Creep deformation tends to be dominant in nature due to stress stabilization. A deformation mechanism map gives a picture of the deformation mechanisms and their relevance. For engineering purposes, dislocation creep (DC) and fluid-assisted diffusional transfer (FADT) creep are important and are the basis for the model developed here. An idealized geometry permits to obtain macroscopic laws for crushed salt from power law (at constant temperature shear strain rate is proportional to a power of shear stress) and Fick's law (dissolved salt diffusion law) which can be considered as basic physical laws. The developed macroscopic laws are generalized to viscoplasticity and viscoelasticity, respectively, for DC and FADT and the creep model compared with experimental data. A further development of the model consists in the coupling of a viscoplastic component that is responsible for mechanisms of 

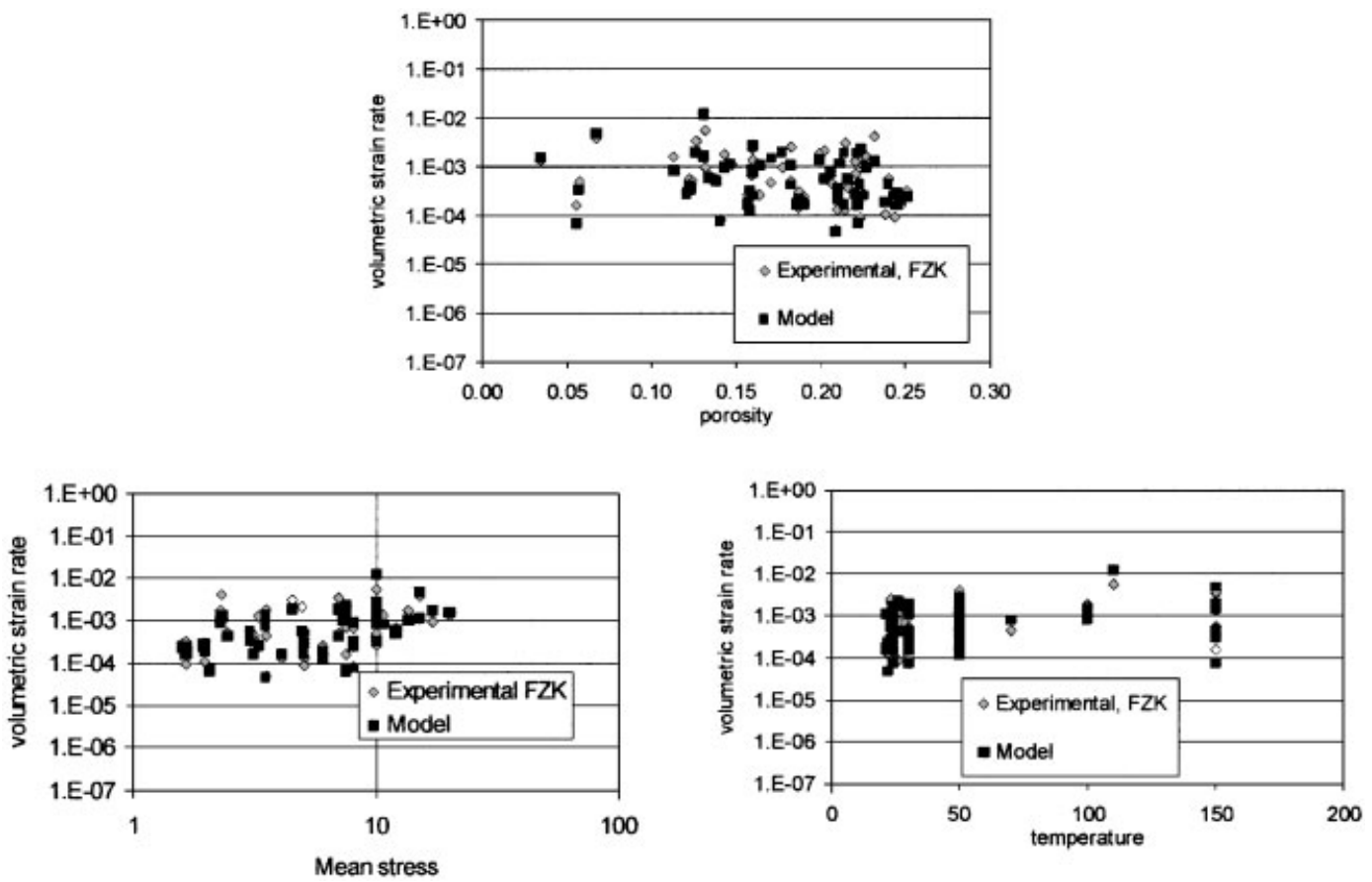

Figure 20. Volumetric strain rate predicted and measured for a series of points (62 sets of $\left(T\left({ }^{\circ} \mathrm{C}\right), \phi\right.$ (dimensionless), $\left.\left.\sigma(\mathrm{MPa}), \mathrm{d} \varepsilon / \mathrm{d} t\left(\mathrm{~s}^{-1}\right)\right)\right)$ that correspond to true triaxial tests carried out by Korthaus at FZK [31].

deformation related to grain rotation, rearrangement and crushing. The critical state theory is considered for this component but with a viscoplastic form. An important point of the model is that hardening in all components is controlled by volumetric inelastic deformation without distinction of the mechanism that is generating it. For instance, the yield envelope of the viscoplastic critical state component expands at constant stress due to accumulation of volumetric creep deformation.

\section{NOMENCLATURE}

$a$

$A(T)$

$B(T)$

$C$

$d_{0}$

$d$

$D_{\mathrm{m}}$

e

$f, g$

$F$

G intercontact thickness (L)

temperature-dependent parameter for DC $\left(\mathrm{T}^{-1}\left(\mathrm{~F} \mathrm{~L}^{-2}\right)^{-n}\right)$

temperature-dependent parameter for FADT $\left(\mathrm{T}^{-1}\left(\mathrm{~F} \mathrm{~L} \mathrm{~L}^{-2}\right)^{-1} \mathrm{~L}^{3}\right)$

auxiliary function of $\left(T, S_{\ell}, d_{0}\right)\left(\mathrm{T}^{-1}\left(\mathrm{~F} \mathrm{~L}^{-2}\right)^{-1}\right)$

characteristic grain size (L)

grain size (L)

molecular diffusion coefficient for salt $\left(\mathrm{L}^{2} \mathrm{~T}^{-1}\right)$

void ratio (dimensionless)

auxiliary functions of void ratio (dimensionless)

stress function for viscoplastic model $\left(\mathrm{F} \mathrm{L}^{-2}\right)$

flow rule for viscoplastic model $\left(\mathrm{F} \mathrm{L}^{-2}\right)$ 


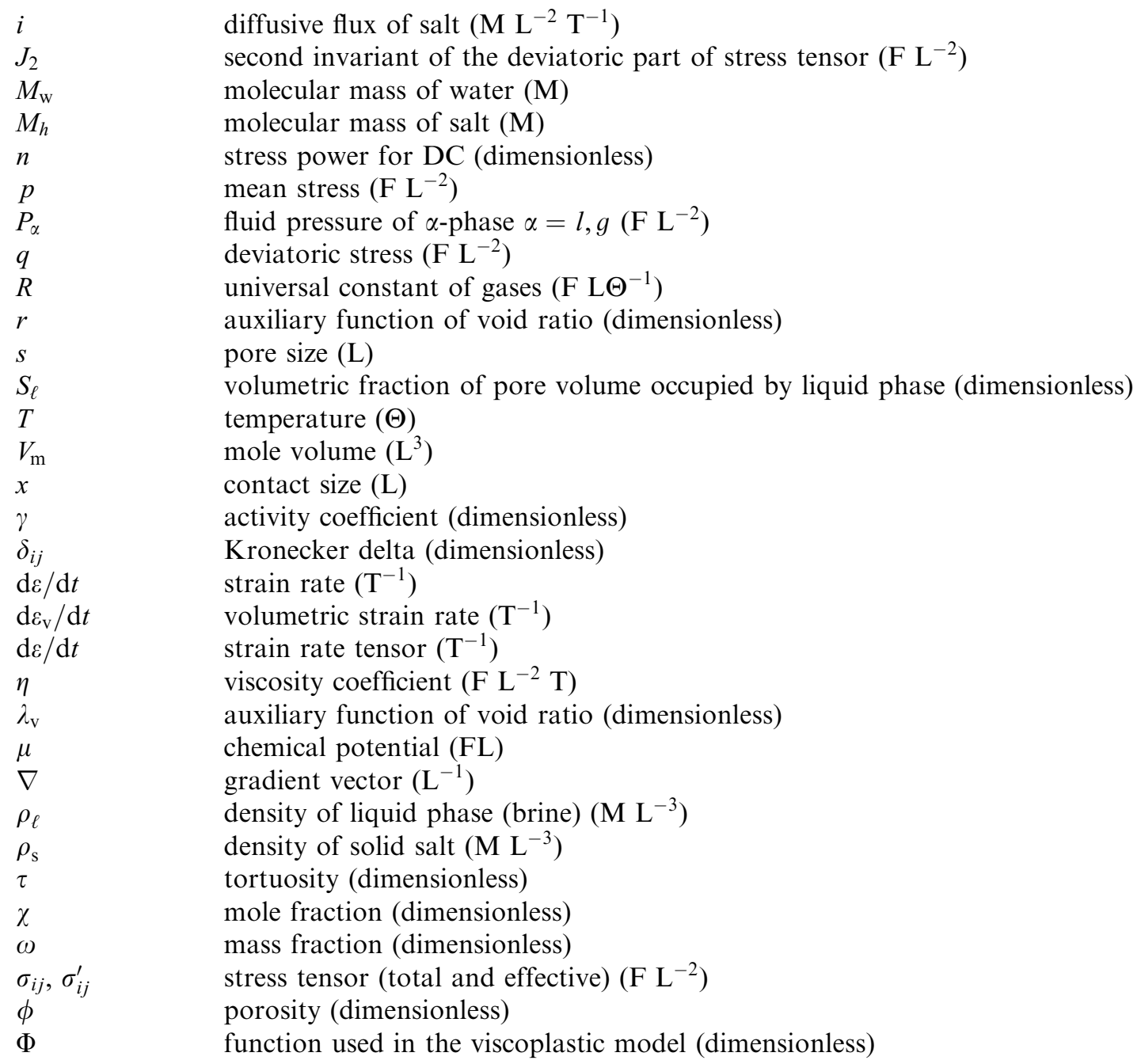

\section{ACKNOWLEDGEMENTS}

The authors would like to acknowledge the contribution to discussions during model development by Jesús Carrera and Eduardo Alonso. This work has been developed in the context of various projects funded by the European Community and ENRESA (Empresa Nacional de Residuos Radioactivos).

\section{REFERENCES}

1. Gens A, Nova R. Conceptual bases for a constitutive model for bonded soils and weak rocks. Proceedings of the International Symposium on Hard Soils \& Soft Rocks, vol. 1. Athens, 1993; 485-494.

2. Lagioia R, Nova R. An experimental and theoretical study of the behaviour of a calacrenite in triaxial compression. Géotechnique 1995; 45:633-648.

3. Kavvadas M, Agnostopoulos A, Kalzetiotis N. A framework for the mechanical behaviour of the cemented Corinth marl. Proceedings of the International Symposium on Hard Soils \& Soft Rocks, vol. 1. Athens, 1993; 577-583.

4. Burghignoli A, Miliziano S, Soccodato FM. Proceedings of the Second International Symposium on Hard Soils \& Soft Rocks, vol. 1. Napoli, 1998; 465-472. 
5. Vaughan PR. Engineering behaviour of weak rocks: some answers and some questions. Proceedings of the International Symposium on Hard Soils \& Soft Rocks, vol. 3. Athens, 1993; 1741-1765.

6. Barton ME. Cohesive sands: the natural transition from sands to sandstones. Proceedings of the International Symposium on Hard Soils \& Soft Rocks, vol. 1. Athens, 1993; 367-374.

7. Olivella S, Carrera J, Gens A, Alonso EE. Porosity variations in saline media caused by temperature gradients coupled to multiphase flow and dissolution/precipitation. Transport in Porous Media 1996; 25:1-25.

8. Olivella S, Carrera J, Gens A, Alonso EE. Non-isothermal multiphase flow of Brine and gas through saline media. Transport in Porous Media 1994; 15:271-293.

9. Munson DE, Dawson PR. Salt constitutive modelling using mechanism maps. The Mechanical Behaviour of Salt I. Pennsylvania State University. Trans. Tech. Publications 1981; 717-737.

10. Farmer IW, Gilbert MJ. Time dependent strength reduction of rock salt. The Mechanical Behaviour of Salt I. Pennsylvania State University, 1981; 3-18.

11. Hunsche U, Albrecht H. Results of true triaxial strength tests on rock salt. Engineering Fracture Mechanics 1990; 35(4/5):867-877.

12. Langer M. The rheological behaviour of rock salt. The Mechanical Behaviour of Salt I. Pennsylvania State University, 1981; 201-240.

13. Cristescu N. Rock Rheology. Kluwer Academic Publishers: Dordrecht, 1989.

14. Liedtke L, Bleich W. Convergence calculations for back-filled tunnels in rock salt. Mechanical Behaviour of Salt II. Transactions of Technical Publications, 1988; 551-580.

15. Kappei G, Gessler K. In situ tests on the behaviour of backfill materials. The Mechanical Behaviour of Salt II. Pennsylvania State University, 1984; 311-328.

16. Spiers CJ, Urai JL, Lister GS, Boland JN, Zwart HJ. The influence of fluid-rock interaction on the rheology of salt rock. Report EUR 10399 EN, Commission of the European Communities, 1986.

17. Spiers CJ, Peach CJ, Brzesowsky RH, Schutjens PMTM, Liezenberg JL, Zwart HJ. Long term rheological and transport properties of dry and wet salt rocks. Report EUR 11848 EN, Commission of the European Communities, 1988.

18. Spiers CJ, Schutjens PMTM, Brzesowsky RH, Peach CJ, Liezenberg JL, Zwart HJ. Experimental determination of constitutive parameters governing creep of rock salt by pressure solution. Geological Society Special Publication No. 54: Deformation Mechanisms, Rheology and Tectonics, 1990; 215:227.

19. Zeuch DH. Isostatic hot-pressing mechanism maps for pure and natural sodium chloride. Applications to nuclear waste isolation in bedded and domal salt formations. International Journal of Rock Mechanics and Mineral Sciences and Geomechanical Abstract 1990; 27(6):505-524.

20. Helle AS, Easterling KE, Ashby MF. Hot-isostatic pressing diagrams: new developments. Acta Metallurgica 1985; 33(12):2163-2174.

21. Olivella S, Gens A, Carrera J, Alonso EE. Behaviour of porous salt aggregates. Constitutive and field equations for a coupled deformation, brine, gas and heat transport model. Mechanical Behaviour of Salt III. Trans Tech Publications, 1993; 269-284.

22. Olivella S. Ph.D. Thesis: 'Nonisothermal multiphase flow of brine and gas through saline media', ETSECCPB, UPC, Barcelona, 1995.

23. Olivella S, Gens A, Carrera J, Alonso EE. Analysis of Creep Deformations of Galleries Backfilled with Porous Salt Aggregates. Mechanical Behaviour of Salt IV. Trans Tech Publications, 1998; 269-284.

24. Schutjens PMTM. Intergranular pressure solution in halite aggregates and quartz sands: an experimental investigation. Thesis, Geologica Ultraiectina, No. 76. Utrecht, 1991.

25. Bird RB, Stewart WE, Lightfoot EN. Transport Phenomena. Wiley: New York, 1960.

26. Lloret A, Alonso EE. Consolidation of unsaturated soils including swelling and collapse behaviour. Geotechnique 1980; 30(4):449-477.

27. Olivella S, Gens A, Alonso EE, Carrera J. Constitutive modelling of porous salt aggregates. In Numerical Models in Geomechanics, vol. 1, Pande, Pietruszczak (eds.). Balkema: Rotterdam, 1992; 179-189.

28. Frost, Ashby. Deformation-Mechanism Maps. Pergamon Press: New York, 1982.

29. Desai CS, Zhang D. Viscoplastic model for geologic materials with generalized flow rule. International Journal for Numerical and Analytical Methods in Geomechanics 1987; 11:603-620.

30. Rothfuchs T, Duijves KA. The HAW project: Demonstration facility for the disposal of high-level waste in salt. Synthesis Report, Nuclear Science and Technology series, EUR 13263 EN, 1991.

31. Bechthold W, Rothfuchs T, Poley A, Ghoreychi M, Heusermann S, Gens A, Olivella S. BAMBUS Project Final Report, EUR 19124EN, 1999.

32. Korthaus E. Consolidation and deviatoric deformation behaviour of dry crushed salt at temperatures up to $150^{\circ} \mathrm{C}$. The Mechanical Behaviour of Salt IV. Trans. Tech. Publications, 1996; 365-378.

33. Fredlund DG, Morgenstern NR. Stress state variables for unsaturated soils. Journal of Geotechnical Engineering Division, ASCE 1977; 103(GT5):447-466.

34. Stührenberg. Untersuchungen zum Materialverhalten von Versatz unter Berücksichtigung der Wechselwirkung Gebirge/Versatz und anderer VarschluBkomponenten in einem Endlager im Salinar, BMFT-Forschungsvorhaben KWA 5801 9, Teilprojekt II, 1990. 